\title{
Cosmological axion and a quark nugget dark matter model
}

\author{
Shuailiang Ge, Xunyu Liang, and Ariel Zhitnitsky \\ Department of Physics and Astronomy, University of British Columbia, Vancouver, \\ British Columbia V6T 1Z1, Canada
}

(Received 4 December 2017; published 13 February 2018)

\begin{abstract}
We study a dark matter (DM) model offering a very natural explanation of two (naively unrelated) problems in cosmology: the observed relation $\Omega_{\mathrm{DM}} \sim \Omega_{\mathrm{visible}}$ and the observed asymmetry between matter and antimatter in the Universe, known as the "baryogenesis" problem. In this framework, both types of matter (dark and visible) have the same QCD origin, form at the same QCD epoch, and are proportional to one and the same dimensional parameter of the system, $\Lambda_{\mathrm{QCD}}$, which explains how these two naively distinct problems could be intimately related, and could be solved simultaneously within the same framework. More specifically, the DM in this model is composed by two different ingredients: the (wellstudied) DM axions and the (less-studied) quark nuggets made of matter or antimatter. We focus on the quantitative analysis of the relation between these two distinct components contributing to the dark sector of the theory determined by $\Omega_{\mathrm{DM}} \equiv\left[\Omega_{\mathrm{DM}}\right.$ (nuggets) $+\Omega_{\mathrm{DM}}$ (axion)]. We argue that the nuggets' DM component always traces the visible matter density, i.e., $\Omega_{\mathrm{DM}}$ (nuggets) $\sim \Omega_{\text {visible }}$, and this feature is not sensitive to the parameters of the system such as the axion mass $m_{a}$ or the misalignment angle $\theta_{0}$. It should be contrasted with conventional axion production mechanisms due to the misalignment when $\Omega_{\mathrm{DM}}$ (axion) is highly sensitive to the axion mass $m_{a}$ and the initial misalignment angle $\theta_{0}$. We also discuss the constraints on this model related to the inflationary scale $H_{I}$, nonobservation of the isocurvature perturbations and the tensor modes. We also comment on some constraints related to various axion search experiments.
\end{abstract}

DOI: 10.1103/PhysRevD.97.043008

\section{INTRODUCTION AND MOTIVATION}

The idea that dark matter may take the form of composite objects of standard model quarks in a novel phase goes back to quark nuggets [1], strangelets [2], nuclearities [3], see also review [4] with a large number of references on the original results. In the models [1-4] the presence of a strange quark stabilizes the quark matter at sufficiently high densities allowing strangelets being formed in the early Universe to remain stable over cosmological time scales. There were a number of problems with the original idea ${ }^{1}$ and we refer to the review paper [4] for the details.

\footnotetext{
${ }^{1}$ In particular, the first order phase transition is a required feature of the system for the strangelet to be formed during the QCD phase transition. However it is known by now that the QCD transition is a crossover rather than the first order phase transition as the recent lattice results [5] unambiguously show. Furthermore, the strangelets will likely evaporate on the Hubble time scale even if they had been formed [6].

Published by the American Physical Society under the terms of the Creative Commons Attribution 4.0 International license. Further distribution of this work must maintain attribution to the author(s) and the published article's title, journal citation, and DOI. Funded by SCOAP ${ }^{3}$.
}

The quark nugget model advocated for in [7] is conceptually similar, with the nuggets being composed of a high density color superconducting (CS) phase. An additional stabilization factor in the quark nugget model is provided by the axion domain walls which are copiously produced during the QCD transition. ${ }^{2}$ The only new additional crucial element in the proposal [7] is that the nuggets could be made of matter as well as antimatter in this framework, see original papers $[7,8]$.

This novel crucial element of the model $[7,8]$ completely changes the entire framework because the dark matter density $\Omega_{\text {dark }}$ and the baryonic matter density $\Omega_{\text {visible }}$ now become intimately related to each other and proportional to each other,

\footnotetext{
${ }^{2}$ In this case the first order phase transition is not required for the nuggets to be formed as the axion domain wall plays the role of the squeezer. Furthermore, the argument related to the fast evaporation of the strangelets as mentioned in footnote 1 is not applicable to the quark nugget model [7] because the vacuum ground state energies inside (CS phase) and outside (hadronic phase) the nuggets are drastically different. Therefore these two systems can coexist only in the presence of the additional external pressure provided by the axion domain wall, in contrast to strangelet models $[1,4]$ which must be stable at zero external pressure.
} 


$$
\Omega_{\text {dark }} \sim \Omega_{\text {visible }},
$$

as will be explained in Sec. II. In other words, the nature of dark matter and the problem of the asymmetry between matter and antimatter in the Universe, normally formulated as the so-called baryogenesis problem, become two sides of the same coin in this framework. To reiterate this claim: The relation (1) is a very generic outcome of the framework, and it is not sensitive to any specific details of the model.

The proposal $[7,8]$ represents an alternative to a baryogenesis scenario when the "baryogenesis" is actually a charge separation process in which the global baryon number of the Universe remains zero. In this model the unobserved antibaryons come to comprise the dark matter in the form of dense antinuggets in the CS phase. The dense nuggets in the CS phase also present in the system such that the total baryon charge remains zero at all times during the evolution of the Universe. The detail mechanism of the formation of the nuggets and antinuggets has been recently developed in Refs. $[9,10]$, and the present work can be considered a continuation of these studies. We review the basics elements of this proposal, its predictions, and the observational consequences including presently available constraints, in Sec. II. We highlight in a few paragraphs below the main questions which will be addressed in the present work.

Our first paper [9] devoted to the formation of the nuggets was focused on the dynamics of a single nugget, its formation, and its time evolution. If the Universe were symmetric during the formation time, an equal number of nuggets and antinuggets would form. However, the coherent (on the enormous scale of the Universe) $C P$-odd axion field was present in the system. Precisely this asymmetry generates the preferential evolution of the system such that the numbers of nuggets and antinuggets would not be identically the same, which was the main result of our paper [10]. As the total baryon charge is conserved in the system, the remaining baryon charge (not hidden in the form of the nuggets and antinuggets) represents the visible baryonic matter contributing to $\Omega_{\text {visible. }}$. This represents a qualitative explanation of generic consequence of this entire framework as represented by (1).

However, in the previous papers $[9,10]$ our main objective was to understand a novel fundamental phenomenon on a qualitative level by simplifying the system itself and neglecting a large number of specific features of the system which may produce quantitative effects of order 1, but cannot change the qualitative picture advocated for in $[9,10]$.

Indeed, in our previous studies we ignored a number of effects. In particular: (a) We assumed that the energy per baryon charge is the same for hadronic and CS phases; (b) we ignored the contribution of the axion domain wall surrounding the quark nugget in CS phase using a simple, order of magnitude estimate for the mass of the nugget,
$M_{\text {nugget }} \sim m_{p} B$; (c) we neglected the contribution of the conventional propagating axions produced by the misalignment mechanism (or by decay of the topological defects) by assuming that $\Omega_{\text {dark }}$ is saturated by the nuggets. All these simplifications allowed us to relate $\Omega_{\text {visible }}$ and $\Omega_{\text {dark }}$ in terms of a single parameter $c$ defined as a ratio of the hidden baryon charge in nuggets and antinuggets, see precise definition in Eq. (4).

The main goal of the present work is to take into account all these complications and numerical factors and incorporate them into a new equation which would be generalization of Eq. (4). We think that the timing for this task is quite appropriate due to a number of reasons. First, there are a number of groups searching for the axion, see the relevant references in next section. If the axion is found with definite mass $m_{a}$ it may not necessarily saturate the entire dark matter density $\Omega_{\mathrm{DM}}$. A finite portion of $\Omega_{\mathrm{DM}}$ could be related to the nuggets' contribution which, according to Refs. [9,10], always accompanies conventional axion production. We refer to the original papers [11-13] and recent reviews [14-22] on the axion dynamics and recent axion searches.

Our goal is to relate these two complementary mechanisms of the axion dynamics: Some portion of the axion field will radiate the propagating axions, while another portion of the coherent axion field will lead to the nuggets' formation. These two mechanisms accompany each other and we want to establish a quantitative relation between the two.

Secondly, it has been recently claimed that the nonobservation of the isocurvature perturbations and the tensor modes imposes strong constraints on the relation between the axion parameter $f_{a}$ and inflation scale $H_{I}$. Our comment here is that such constraints are normally imposed by assuming that the relic axions saturate the dark matter (DM) density. However, if the nuggets become the dominant contributor to $\Omega_{\mathrm{DM}}$, the corresponding constraint drastically weakens because the axions' contribution to $\Omega_{\mathrm{DM}}$ becomes subdominant, and as a consequence, the isocurvature perturbations will be suppressed for the same axion mass $m_{a}$ and coupling $f_{a}$. These few comments suggest that there are a number of relevant parameters in the theory which crucially depend on the relative contribution of the relic axions versus nuggets' portion to $\Omega_{\mathrm{DM}}$. The goal of this work is to elaborate on those relations.

The paper is organized as follows. In Sec. II we take an overview of the basic idea and phenomenological consequences on the proposal $[7,8]$ with emphasis how the baryogenesis is replaced by the charge separation effect $[9,10]$. In Sec. III, we will introduce our notations and definitions of the parameters to be computed. In Sec. IV, we will focus on the internal structure of the nuggets. In particular, we compute the energy per baryon charge of a single stabilized (anti)nugget at zero temperature. Then in Sec. V, using the results of previous two sections, we 
produce a number of numerical plots and show that there is a large region of parametrical space in this model, which is consistent with all presently available constraints. The corresponding constraints have been obtained from a variety of independent astrophysical, cosmological, satellite, and ground based observations. Furthermore, we will show that this model is also consistent with known constraints from the axion search experiments. In Sec. VI, we briefly summarize the main results of this paper and discuss the potential future directions. In particular, we speculate that unexpected correlations between the frequency of appearance of the solar flares and the intensity of the dark matter fluxes observed on the Sun might be related to the antiquark nuggets studied in this work.

\section{AXION QUARK NUGGET (AQN) MODEL}

The AQN model in the title of this section stands for the axion quark nugget model to emphasize an essential role of the axion field and avoid confusion with earlier models such as the quark nuggets, strangelets, and nuclearities mentioned in the Introduction.

The basic ideas of the original proposal $[7,8]$ can be explained as follows: It is commonly assumed that the Universe began in a symmetric state with zero global baryonic charge and later (through some baryon number violating process, so-called baryogenesis) evolved into a state with a net positive baryon number. As an alternative to this scenario we advocate a model in which the baryogenesis is actually a charge separation process in which the global baryon number of the Universe remains zero. In this model the unobserved antibaryons come to comprise the dark matter in the form of dense nuggets of quarks and antiquarks in the CS phase. The formation of the nuggets made of matter and antimatter occurs through the dynamics of shrinking axion domain walls, see original papers $[9,10]$ for the technical details.

If the fundamental $\theta$ parameter of QCD were identically zero during the formation time, equal numbers of nuggets made of matter and antimatter would be formed. However, the fundamental $C P$ violating processes associated with the $\theta$ term in QCD result in the preferential formation of antinuggets over nuggets. This source of strong $C P$ violation is no longer available at the present epoch as a result of the axion dynamics when $\theta$ eventually relaxes to zero, see Fig. 1. As a result of these $C P$ violating processes the number of nuggets and antinuggets being formed would be different. This difference is always on the order of one effect irrespective to the parameters of the theory, the axion mass $m_{a}$, or the initial misalignment angle $\theta_{0}$, as argued in $[9,10]$. As a result of this disparity between nuggets and antinuggets a similar disparity would also emerge between visible quarks and antiquarks. Precisely this disparity between visible baryons and antibaryons eventually leads (as a result of the annihilation processes) to the system

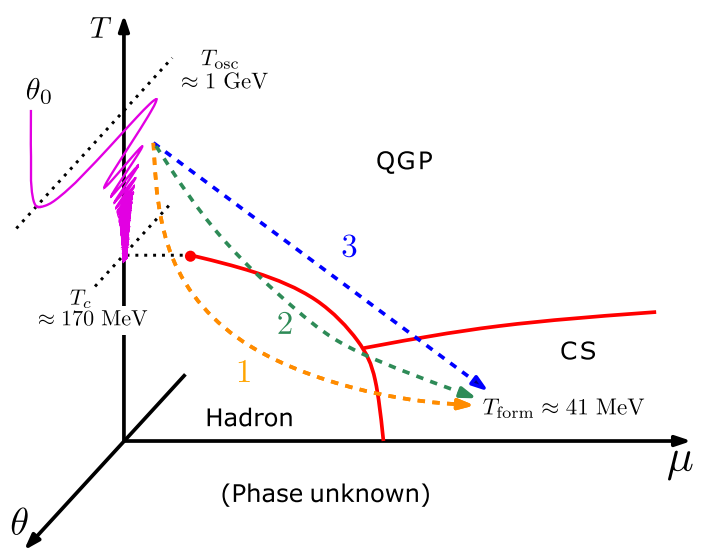

FIG. 1. This diagram illustrates the interrelation between the axion production due to the misalignment mechanism and the nuggets' formation which starts before the axion field $\theta$ relaxes to zero. Possible cooling paths are denoted as path 1, 2 or 3 . The phase diagram is in fact much more complicated as the dependence on the third essential parameter, the $\theta$ is not shown as it is largely unknown. It is assumed that the initial axion field starts at $\theta_{0}$, while the nuggets complete their formation in the CS region at $T_{\text {form }} \approx 41 \mathrm{MeV}, \mu>\mu_{c}$ and $\theta \approx 0$.

when exclusively one species of visible baryons remain in the system, in agreement with observations.

We illustrate this interrelation between the axion dynamics and the nuggets' formation in Fig. 1 when both processes occur during the same QCD epoch. The key element of the proposal is the presence of the coherent axion $\theta(t)$ field, which continues to oscillate when the nuggets' formation starts. The time evolution of the nuggets continues until low temperature $T_{\text {form }}$ when the axion field assumes its final destination $\theta=0$, which is observed today. However, the originally produced asymmetry between nuggets and antinuggets cannot be washed out, as explained in [10].

This disparity between nuggets and antinuggets unambiguously implies that the total number of antibaryons will be less than the number of baryons in early Universe plasma. This is precisely the reason why the resulting visible and dark matter densities must be the same order of magnitude (1) in this framework as they are both proportional to the same fundamental $\Lambda_{\mathrm{QCD}}$ scale, and they both are originated at the same QCD epoch. If these processes are not fundamentally related, the two components $\Omega_{\text {dark }}$ and $\Omega_{\text {visible }}$ could easily exist at vastly different scales.

Another fundamental ratio is the baryon to entropy ratio at the present time,

$$
\eta \equiv \frac{n_{B}-n_{\bar{B}}}{n_{\gamma}} \simeq \frac{n_{B}}{n_{\gamma}} \sim 10^{-10} .
$$

In our proposal (in contrast to conventional baryogenesis frameworks) this ratio is determined by the formation temperature $T_{\text {form }} \simeq 41 \mathrm{MeV}$ at which the nuggets and 
antinuggets complete their formation. We note that $T_{\text {form }} \approx \Lambda_{\mathrm{QCD}}$. This temperature is determined by the observed ratio (2). The $T_{\text {form }}$ assumes a typical QCD value, as it should, as there are not any small parameters in QCD, see Fig. 1.

Unlike conventional dark matter candidates, such as WIMPs (weakly interacting massive particles), the dark matter/antimatter nuggets are strongly interacting and macroscopically large, nuclear density objects with a typical size $\left(10^{-5}-10^{-4}\right) \mathrm{cm}$, and a baryon charge which ranges from $B \sim 10^{23}$ to $B \sim 10^{28}$. However, they do not contradict any of the many known observational constraints on dark matter or antimatter for three main reasons [23]:

(a) They carry a very large baryon charge $\left(|B|>10^{23}\right)$ which is determined by the size of the nugget $\left(\sim m_{a}^{-1}\right)$. As a result, the number density of the nuggets is very small $\left(\sim B^{-1}\right)$. Their interaction with visible matter is thus highly suppressed. In particular, the quark nuggets essentially decouple from cosmic microwave background $(\mathrm{CMB})$ photons, and therefore, they do not destroy the conventional picture for the structure formation.

(b) The nuggets have a huge mass $M_{\text {nugget }} \sim m_{p} B$, therefore the effective interaction is very small $\left(\sigma / M_{\text {nugget }} \sim 10^{-10} \mathrm{~cm}^{2} / \mathrm{g}\right)$, which is evidently well below the upper limit of the conventional DM constraint $\sigma / M_{\mathrm{DM}}<1 \mathrm{~cm}^{2} / \mathrm{g}$.

(c) The quark nuggets have a very large binding energy due to the large gap $(\Delta \sim 100 \mathrm{MeV})$ in the CS phase. Therefore, the strongly bound baryon charge is unavailable to participate in the big bang nucleosynthesis at $T \approx 1 \mathrm{MeV}$, long after the nuggets had been formed.

We emphasize that the weakness of the visible-dark matter interaction in this model is due to a small geometrical parameter $\sigma / M \sim B^{-1 / 3}$ which replaces the conventional requirement of sufficiently weak interactions for WIMPs.

In this short overview of the model we also want to make a few comments regarding the observational consequences of the proposal. It is known that the galactic spectrum contains several excesses of diffuse emission, the origin of which is uncertain, the best known example being the strong galactic $511 \mathrm{keV}$ line. Our comment here is that this model offers a potential explanation for several of these diffuse components (including the $511 \mathrm{keV}$ line and the accompanying continuum of $\gamma$ rays in the $100 \mathrm{keV}$ and few $\mathrm{MeV}$ ranges, as well as $\mathrm{x}$-rays, and radio frequency bands). For further details see the original works [24-30] with specific computations in different frequency bands in galactic radiation, and a short overview [31].

As we already mentioned, the typical size of a nugget $\sim m_{a}^{-1}$ is determined by the axion mass, yet to be discovered. Therefore, a typical baryon charge of the nuggets and their size distribution are also unknown characteristics of the model. However, it is important to emphasize that the allowed window for the axion mass $10^{-6} \mathrm{eV} \leq m_{a} \leq$ $10^{-2} \mathrm{eV}$ corresponds to the range of the nuggets' baryon charge $B$ which largely overlaps with all presently available and independent constraints on such dark matter masses and baryon charges,

$$
10^{23} \leq|B| \leq 10^{28}
$$

see e.g. $[31,32]$ for a review. ${ }^{3}$ To reiterate the same claim: The allowed window (3) for the baryon charge $B$ is perfectly consistent with all presently available constraints.

Furthermore, if one takes the recent conjecture [33,34] that the extreme ultraviolet (EUV) and soft x-ray emission from the solar corona are due to the antiquark nuggets hitting the Sun from outer space, then one can make one step further and identify the observed "nanoflares" with annihilation events of the antinuggets in the solar corona. Taking this identification literally one can translate the observed energy distribution of the nanoflares in the solar corona in terms of the nuggets' baryon charge distribution. This identification leads to an independent estimate on possible values for $B$ which is perfectly consistent with the allowed range (3) for the baryon charge of the nuggets $[33,34]$. Therefore, in the analysis which follows we treat the window (3) as the solid constraint on the allowed magnitude of the nuggets' baryon charge.

\section{THE AQN MODEL: NOTATIONS AND DEFINITIONS}

In this section, we will first briefly introduce our notations for the AQN model, which are constituted by two parts: One portion is represented by the nuggets and antinuggets, while the other component is represented by free propagating axions which were produced by the axion field (through e.g. the misalignment mechanism). In order to quantitatively describe this model, we have to derive a general expression which relates these two complementary contributions to the $\Omega_{\mathrm{DM}}$, as they both originated from the one and the same axion field during the same QCD epoch as illustrated in Fig. 1.

To proceed with this task we first recall a simplified expression derived in our previous papers $[9,10]$ where a

\footnotetext{
${ }^{3}$ The smallest nuggets with $B \sim\left(10^{23}-10^{24}\right)$ naively contradict the constraints cited in [32]. However, the corresponding constraints are actually derived with the assumption that nuggets with a definite mass (smaller than $55 \mathrm{~g}$ ) saturate the dark matter density. In contrast, we assume that the peak of the nuggets' distribution corresponds to a larger value of mass, $\langle B\rangle \geq 10^{25}$, while the small nuggets represent a tiny portion of the total dark matter density. The same comment also applies to the larger masses excluded by Apollo data as reviewed in [31]. Large nuggets with $B \sim 10^{28}$ may exist, but represent a small portion of the total dark matter density, and therefore, do not contradict the Apollo's constraints, see also some comments on the baryon charge distribution (34) in Sec. VI.
} 
number of effects have been neglected, as mentioned in Sec. I. In the simplified treatment we characterized the system by a single crucial parameter $c$ which describes the disparity between nuggets and antinuggets and is defined as follows :

$$
\Omega_{\text {dark }} \simeq\left(\frac{1+c}{1-c}\right) \Omega_{\text {visible }}, \quad c \equiv \frac{\left|B_{\text {nuggets }}\right|}{\left|B_{\text {antinuggets }}\right|} .
$$

The most important consequence of this model is given by relation (1). It is expressed now in terms of numerical parameter $c$ as Eq. (4) states. As we have already stated, this equation represents a very generic consequence of the entire framework. The parameter $0<c<1$ is obviously positive and cannot be greater than 1 as Eq. (4) suggests. This is because the baryon charge hidden in the nuggets should be less than the baryon charge hidden in the antinuggets, as the difference represents the baryon charge of the visible matter in the hadronic phase we presently observe.

As we already mentioned in the Introduction, in our previous studies $[9,10]$ the relation (4) is not accurate as we previously ignored a number of numerical factors. In particular, we assumed that the energy per baryon charge for hadronic and CS phases is the same; we ignored the contribution of the axion domain wall surrounding the quark nugget in the CS phase; we neglected the contribution of the conventional propagating axions produced by the misalignment mechanism (or by decay of the topological defects) by assuming that $\Omega_{\text {dark }}$ is saturated by the nuggets. All these simplifications allowed us to derive a simple formula (4) in terms of a single parameter $c$, which is very important for qualitative, but not quantitative, arguments. Now we want to generalize relation (4) by accounting for all these (previously neglected) effects. The corresponding modifications obviously do not affect the basic qualitative claim (1), but may change some numerical factors, which is precisely the main objective of the present study.

Traditionally, the axion is regarded as one of the leading candidates for DM, see e.g. recent reviews [20-22] and the references therein. It is normally assumed that the initial coherent axion field radiates the propagating axions through the misalignment mechanism or as a result of decay of the topological defects.

However, these two distinct processes of the axion's field relaxation will be always accompanied by another complementary mechanism (related to the so-called $N_{\text {DW }}=1$ axion domain walls), as recently advocated for in Refs. [9,10]. The corresponding scenario is given an overview in Secs. I and II, and shall not be elaborated on

\footnotetext{
${ }^{4}$ The definition of $c$ in the present work is different than previous studies $[9,10]$, where $c \equiv B_{\text {antinuggets }} / B_{\text {nuggets }}$ was negative and inversely defined, in contrast to (4).
}

here. The only important elements to be mentioned now are (a) this scenario assumes that the Peccei-Quinn (PQ) symmetry breaking occurs before or during inflation, and (b) this scenario leads to a very generic consequence (1) which holds irrespective of any parameters of the system such as $m_{a}$ or initial misalignment angle $\theta_{0}$. This generic prediction of this new proposal should be contrasted with two other well-known and well-studied mechanisms of the axion field's relaxation when the corresponding contribution to the DM density is highly sensitive to the axion parameters, $\Omega_{\text {dark }} \sim \theta_{0}^{2} / m_{a}^{7 / 6}$.

We now proceed with our definitions and notations of the AQN model, which incorporates both contributions: the conventional axion production reviewed in [20-22] and the nuggets' contribution studied in $[9,10]$. As usual, we use the ratio of different component densities to critical density today to mark their proportions,

$$
\Omega_{i}=\rho_{i} / \rho_{\mathrm{cr}}, \quad i=b, \pm, a,
$$

where label $i$ stands for different species: $b$ for baryon; + for nugget and - for antinugget; and $a$ for free axions. $\rho_{\mathrm{cr}}$ is the critical density of the Universe today. Then we have

$$
\Omega_{\mathrm{DM}}: \Omega_{b}=\left(\Omega_{+}+\Omega_{-}+\Omega_{a}\right): \Omega_{b} \approx 5: 1,
$$

where $\Omega_{\mathrm{DM}}$ is represented by two parts, the axion contribution $\Omega_{\mathrm{DM}}($ axion $) \equiv \Omega_{\mathrm{a}}$ and the nuggets' portion $\Omega_{\mathrm{DM}}($ nugget $) \equiv\left(\Omega_{+}+\Omega_{-}\right)$.

Next, to describe the difference between nugget and antinugget, we define the following parameters:

$$
c_{\Omega} \equiv \frac{\Omega_{+}}{\Omega_{-}}=\frac{N_{+} E_{+}}{N_{-} E_{-}}=\frac{N_{+} \epsilon_{+} B_{+}}{N_{-} \epsilon_{-} B_{-}}=\frac{N_{+}}{N_{-}} c_{\epsilon} \cdot c,
$$

where

$$
c_{\epsilon} \equiv \frac{\epsilon_{+}}{\epsilon_{-}}, \quad c \equiv \frac{B_{+}}{B_{-}}, \quad 0<c<1,
$$

and $N_{ \pm}$is the number density of (anti)nuggets in the Universe today; $E_{ \pm}, B_{ \pm}$, and $\epsilon_{ \pm}=E_{ \pm} / B_{ \pm}$are, respectively, the energy, baryon number, and energy density per baryon charge for a single nugget or antinugget. In these relations both baryon charges $B_{+}$and $B_{-}$are defined to be positive numbers. The definition of $c$ in (8) coincides with the definition given in (4).

As we already mentioned after Eq. (4) we neglected a number of important numerical factors in our previous studies $[9,10]$. We can now formalize these effects in a very precise way using our definitions (7) and (8). In particular, $\epsilon_{ \pm}$is the energy per baryon charge in the CS phase is not the same as in hadronic phase, i.e., $\epsilon_{ \pm} \neq m_{p} / 3$. Furthermore, $E_{ \pm}$, which was previously estimated as $E_{+}=E_{-}=B m_{p}$, now includes the contribution from 
the surrounding axion domain wall, and obviously has a much more complicated structure. In addition, we previously ignored the contribution from free propagating axions by assuming $\Omega_{a}=0$. All these new elements will be incorporated in our analysis in Sec. IV.

To simplify our analysis we want to make a technical assumption that $N_{+} \simeq N_{-}$in Eq. (7). It does not affect any of our conclusions, as we argue below. The basic justification for this assumption is as follows: The initial distributions of the nuggets and antinuggets are the same. It is just their evolution in the background of the coherent axion $C P$-odd field generates the asymmetry between them, as argued in [10]. As time evolves the nuggets and antinuggets assume different size distribution, but their number densities, $N_{+}$ and $N_{-}$, should stay the same. ${ }^{5}$ This information is coded in Eqs. (7) and (8) by the relation $B_{+} \neq B_{-}$.

Substituting $N_{+}=N_{-}$into Eq. (7) we arrive at the desired expression,

$$
c_{\Omega}=c_{\epsilon} \cdot c .
$$

This equation has obvious physical meaning and essentially states that the relative contribution of the nuggets and antinuggets to $\Omega_{\mathrm{DM}}$ is proportional to the corresponding baryon charges expressed in terms of parameter $\sim c$, and it is also proportional to the difference of their energy densities parametrized by $c_{\epsilon}$.

Now we want to derive an equation, similar to (4), which accounts for a number of the effects which were previously ignored, including the differences between nuggets and antinuggets. With this purpose we express the baryon number conservation in the following form:

$$
B_{-}-B_{+} \simeq \frac{1}{N_{ \pm}} \frac{3 \rho_{b}}{m_{p}}
$$

where $m_{p}$ is the mass of a single baryon charge and can be approximated by the proton mass. The coefficient 3 in Eq. (10) corresponds to our normalization of the baryon charge in the present work. This normalization is consistent with our definition of $\mu$ corresponding to the quark (rather than baryon) chemical potential. Furthermore, $B_{ \pm}$in all our formulas are positive numbers, as mentioned above. Therefore, the $B_{ \pm}$parameters count for the number of quarks in the system, rather then their baryon charges.

With these comments in mind and using Eqs. (5), (7), and (10), we arrive at the following relations:

\footnotetext{
${ }^{5}$ There is some loophole in this argument as smaller bubbles may completely collapse, while larger bubbles survive the evolution. As the size distribution for $B_{-}$is different than $B_{+}$ the corresponding number densities for $N_{+}$and $N_{-}$could also be different as a result of different rates for collapsed nuggets. We expect that this effect is quite small. In any event, this effect can be easily accommodated by redefinition of the effective $B_{+}^{\text {eff }}$ and $B_{-}^{\text {eff }}$ values accounting for the collapsed nuggets.
}

$$
\frac{\Omega_{+}}{\epsilon_{+}}\left(\frac{1}{c}-1\right)=\frac{\Omega_{-}}{\epsilon_{-}}(1-c)=\frac{3 \Omega_{b}}{m_{p}} .
$$

The coefficient $c \in(0,1)$ in these relations satisfies the same constraint as in our simplified treatment of the problem presented in (4). Therefore,

$$
0<c<1 \text {. }
$$

Our next step is to rewrite Eqs. (6) and (7) in the following convenient form:

$$
\Omega_{+}=c_{\Omega} \cdot \frac{\Omega_{\mathrm{DM}}-\Omega_{a}}{1+c_{\Omega}}, \quad \Omega_{-}=\frac{\Omega_{\mathrm{DM}}-\Omega_{a}}{1+c_{\Omega}} .
$$

The last step to achieve our goal is to use Eqs. (11) and (9) to arrive at the final expressions which will be used in our numerical studies in Sec. V:

$$
\begin{gathered}
\frac{1-c}{1+c_{\epsilon} c}=\frac{3 \epsilon_{-}}{m_{p}} \cdot \frac{\Omega_{b}}{\Omega_{\mathrm{DM}}-\Omega_{a}}, \\
c_{\epsilon} \cdot \frac{1-c}{1+c_{\epsilon} c}=\frac{3 \epsilon_{+}}{m_{p}} \cdot \frac{\Omega_{b}}{\Omega_{\mathrm{DM}}-\Omega_{a}} .
\end{gathered}
$$

Equation (14) is a generalization of our previous simplified expression (4). It accounts for a number of numerical effects mentioned previously. Formula (14) obviously reduces to our previous expression (4) in the limit when $\Omega_{a}=0$ and nuggets and antinuggets have the same energies, i.e., $\epsilon_{+}=\epsilon_{-}=m_{p} / 3$ such that $c_{\epsilon}=1$.

While the numerical estimates for parameters $3 \epsilon_{ \pm} / m_{p}$ and $c_{\epsilon}$ entering (14) will be discussed in detail in Sec. IV, the rest of this section is devoted to a short overview of known estimates of the parameter $\Omega_{a}$ which also enters Eq. (14).

The corresponding computations of $\Omega_{a}$ have been carried out in a number of papers. In what follows we limit ourselves by reviewing the estimates of $\Omega_{a}$ resulted from the misalignment mechanism [35], while leaving out the contribution related to the decay of the topological defects. ${ }^{6}$ We emphasize that we do not exclude the corresponding contributions related to the topological defects because they are unimportant. Rather, we omit them because their role is largely unknown under present circumstances when the PQ

\footnotetext{
${ }^{6}$ There are a number of uncertainties and remaining discrepancies in the corresponding estimates. We shall not comment on these subtleties by referring to the original papers [36-40]. According to the most recent computations presented in Ref. [40], the axion contribution to $\Omega_{\mathrm{DM}}$ as a result of decay of the topological objects can saturate the observed DM density today if the axion mass is in the range $m_{a}=(2.62 \pm 0.34) \times 10^{-5} \mathrm{eV}$, while the earlier estimates suggest that the saturation occurs at a larger axion mass. One should also emphasize that the computations [36-40] have been performed with assumption that PQ symmetry was broken after inflation.
} 
symmetry is broken before/during inflation. ${ }^{7}$ In addition, even in a different scenario when the PQ symmetry is broken after inflation, the question of whether it saturates the observed dark matter density remains controversial, as mentioned in footnote 6 . Thus, we leave out this contribution to simplify our notations and our analysis as the focus of the present work is the nuggets' contribution to $\Omega_{\mathrm{DM}}$ rather than the direct axion production represented by $\Omega_{a}$. In other words, $\Omega_{a}$ contribution is kept in our formulas for normalization purposes to illustrate the significance (or insignificance) of the nuggets' contribution to $\Omega_{\mathrm{DM}}$ as a function of parameters. The contribution to the $\Omega_{a}$ due to the decay of the topological objects can be always incorporated into our formulas once the uncertainties of this contribution are better understood.

For the vacuum misalignment production of free propagating axions, we adopt the general formula as presented in Ref. [20],

$$
\begin{aligned}
\Omega_{a} h^{2} \approx & 2 \times 10^{4}\left(\frac{f_{a}}{10^{16} \mathrm{GeV}}\right)^{7 / 6}\left(\theta_{a, i}^{2}+\frac{H_{I}^{2}}{\left(2 \pi f_{a}\right)^{2}}\right) \\
& \times F_{\mathrm{anh}}\left(\sqrt{\theta_{a, i}^{2}+\frac{H_{I}^{2}}{\left(2 \pi f_{a}\right)^{2}}}\right),
\end{aligned}
$$

with

$$
F_{\text {anh }}(x)=\left[\ln \left(\frac{e}{1-x^{2} / \pi^{2}}\right)\right]^{7 / 6},
$$

where $H_{I}$ is the inflationary Hubble scale and $F_{\text {anh }}(x)$ is the correction factor due to the anharmonic cosine part in axion potential $[20,41]$. The parameter $\theta_{a, i}$ in (15) is the initial misalignment angle and $H_{I} /\left(2 \pi f_{a}\right)$ is the backreaction contribution to this homogeneous field displacement due to the isocurvature perturbations. The parameter $f_{a}$ and the axion mass $m_{a}$ are not independent parameters, as their product is fixed by the topological susceptibility of QCD, $\chi=f_{a}^{2} m_{a}^{2}$. Using the recent value $\chi=0.0216 \mathrm{fm}^{-4}$ at zero temperature [42], we have

$$
m_{a} \simeq 5.7 \times 10^{-4} \mathrm{eV}\left(\frac{10^{10} \mathrm{GeV}}{f_{a}}\right)
$$

This completes our short overview of the $\Omega_{a}$ contribution entering our basic formulas (14).

Section IV is mainly devoted to the estimates of $3 \epsilon_{ \pm} / m_{p}$ and $c_{\epsilon}$ which, along with $\Omega_{a}$, also enter our basic Eq. (14). Then in Sec. V, using the results of Secs. III and IV, we do a numerical analysis to study the allowed window and

\footnotetext{
${ }^{7}$ It was explained in [9] in great detail that the so-called $N_{\mathrm{DW}}=$ 1 domain walls must be present in the system even when PQ symmetry was broken before/during inflation, see item 5 in Sec. III of [9]. It should be contrasted with conventional studies on the role of the topological defects when the PQ symmetry is assumed to be broken after the inflation, see also footnote 6 with its related comment.
}

constraints related to the phenomenological parameters of the AQN dark matter model.

We conclude this section with the following generic comment. The nuggets' contribution given by $\Omega_{ \pm}$and the direct axion production represented by $\Omega_{a}$ always accompany each other during relaxation of the dynamical axion field to zero at the QCD epoch. These contributions to $\Omega_{\mathrm{DM}}$ represent complementary mechanisms and cannot be formally separated (e.g. by variation of a free parameter of the system such as $f_{a}$ ), as the closed $N_{\mathrm{DW}}=1$ domain wall bubbles, which are responsible for the nuggets' formation, can be produced irrespective of whether the PQ scale is above or below the inflationary scale $H_{I}$, as argued in [9].

\section{INTERNAL STRUCTURE OF THE NUGGETS}

The main goal of this section is to estimate the energy per baryon charge of a stabilized quark nugget at zero temperature. We consider two drastically different models to accomplish this task. The main reason to consider two different models (constructed on fundamentally different principles) is to test the sensitivity (or nonsensitivity) to different phenomenological parameters effectively describing the strongly coupled QCD. For simplicity and without loss of generality, we assume that the CS phase assumes the simplest possible structure in the form of the color flavor locked (CFL) phase without any additional complications such as possible meson condensation.

The first model largely follows the original work [7]. However, the difference from the previous analysis is that the first paper on the subject [7] was mostly dealing with fundamental and basic questions on principle possibility to stabilize the nuggets by the axion domain walls. The goal of the present study is quite different as we want to produce some quantitative results on the parameters entering the basic Eq. (14).

The model [7] considers the equilibration between the Fermi pressure, the domain wall surface tension, the "bag constant" pressure $\sim E_{B}$, and, finally, the quark-quark interaction related to the CS gap. The energy of a stabilized nugget can be represented in the following form:

$$
\begin{aligned}
E^{(1)}= & 4 \pi \sigma_{\mathrm{eff}} R^{2}+\frac{g \mu^{4}}{6 \pi} R^{3}-\frac{3 \Delta^{2} \mu^{2}}{\pi^{2}} V \\
& +E_{B} \theta\left(\mu-\mu_{1}\right)\left(1-\frac{\mu_{1}^{2}}{\mu^{2}}\right) V, \quad V \equiv \frac{4 \pi}{3} R^{3},
\end{aligned}
$$

while the nuggets' baryon number can be estimated as follows:

$$
B=g V \int_{0}^{\mu} \frac{d^{3} p}{(2 \pi)^{3}}=\frac{2 g}{9 \pi} \mu^{3} R^{3},
$$

where our normalization corresponds to $B=1$ per single quark degree of freedom in order to remain consistent with the notations in Sec. III. 
A few comments are in order. First of all, the "squeezer" parameter $\mu_{1}$ is estimated to be $330 \mathrm{MeV}$, the degeneracy factor for the CFL phase is estimated as $g \simeq 2 N_{c} N_{f} \simeq 18$. Parameters $\mu$ and $R$ entering (18) and (19) are the chemical potential and radius of the nugget, respectively, while the bag constant $E_{B} \simeq(150 \mathrm{MeV})^{4}$ and the CS gap $\Delta \simeq$ $100 \mathrm{MeV}$ assume their commonly accepted magnitudes, see original work [7] with some arguments supporting these numerical values.

The domain wall tension $\sigma_{\text {eff }}$ entering (18) requires some additional comments. First of all, the effective domain wall tension $\sigma_{\text {eff }}$ should not be confused with the conventional surface tension $\sigma \simeq 8 f_{a}^{2} m_{a}$, which normally enters the computations [36-40] of the axion production due to the decay of the topological defects.

There are two main reasons for this important difference. First of all, the axion domain wall solution in our case interpolates between topologically distinct vacuum states in hadronic and CS phases, in contrast to a conventional axion domain wall which interpolates between topologically distinct hadronic vacuum states. The chiral condensate may or may not be formed in the CS phase. It strongly affects the topological susceptibility in the CS phase, which could be much smaller than in the conventional hadronic phase. The well-known manifestation of this difference is the expected smallness of the $\eta^{\prime}$ mass in the CS phase in comparison with the hadronic phase. One should emphasize that the $2 \pi$ periodicity of the axion $(\theta)$ and the NambuGoldstone fields $\left(\eta^{\prime}\right)$ still holds in the presence of the chemical potential $\mu$ in dense matter CS phases [43]. Therefore, the topological reason for mere existence of the axion domain wall still persists, while the numerical value of the tension $\sigma_{\text {eff }}$ will deviate from its conventional expression $\sigma$ computed in the hadronic phase.

The second reason for strong deviation of the $\sigma_{\text {eff }}$ from conventional expression for $\sigma$ is that formula $\sigma \simeq 8 f_{a}^{2} m_{a}$ was derived assuming the thin-wall approximation when the domain wall is assumed to be almost flat, i.e., a typical curvature of the domain wall structure is much smaller than its width. This approximation is obviously badly violated because the axion domain wall width is of order $m_{a}^{-1}$, while typical curvature is comparable with the width of the domain wall as these two parameters are related in our framework, $R \sim m_{a}^{-1}$. The physical consequence of this relation is that the axion field strongly overlaps within the nuggets' volume. This effect is expected to drastically reduce the domain wall tension. ${ }^{8}$

\footnotetext{
${ }^{8}$ The corresponding large modifications can be understood from a simple model when the domain wall is bent, allowing a strong overlap between opposite sides of the wall. The effective domain wall tension obviously receives the modifications as a result of this bending geometry when the axion field configuration deviates from a simple well-known one-dimensional solution.
}

To account for these complicated QCD effects we define $\sigma_{\text {eff }} \equiv \kappa \cdot \sigma$, with an unknown phenomenological parameter $0<\kappa<1$ which accounts for the physics mentioned above. In particular, the violation of the thin-wall approximation was modeled in [9] by introducing a suppression factor $\exp \left(-R_{0} / R_{\text {form }}\right)$. The corresponding suppression could be quite strong and can be as small as $10^{-5}$ assuming a typical formation radius $R_{\text {form }} \sim 0.1 R_{0}$ as studied in [9]. In what follows we treat $\kappa$ as a free phenomenological parameter.

Our goal now is to minimize the expression (18). To achieve this goal we introduce two dimensionless variables $x$ and $\sigma_{0}$ as

$$
\begin{aligned}
x & \equiv R \frac{E_{B}^{1 / 4}}{B^{1 / 3}}=\frac{E_{B}^{1 / 4}}{\mu}\left(\frac{8 \pi}{2 g}\right)^{\frac{1}{3}}, \\
\sigma_{0} & \equiv \frac{\sigma_{\mathrm{eff}}}{B^{1 / 3} E_{B}^{3 / 4}}=\frac{8 \chi}{E_{B}^{3 / 4}} \frac{1}{\kappa^{-1} B^{1 / 3} m_{a}},
\end{aligned}
$$

where we express $\sigma$ in terms of topological susceptibility $\chi=f_{a}^{2} m_{a}^{2}=0.02 \mathrm{fm}^{-4}$. Then, the energy per quark $\epsilon \equiv$ $E / B$ can be expressed as

$$
\begin{aligned}
\epsilon_{\mathrm{tot}}^{(1)}(x) \equiv & \frac{E^{(1)}}{B}=\epsilon_{\mathrm{DW}}+\epsilon_{\mathrm{QCD}}^{(1)}(x), \\
\epsilon_{\mathrm{DW}}(x)= & E_{B}^{1 / 4} 4 \pi \sigma_{0} x^{2}=\frac{32 \pi \chi}{E_{B}^{1 / 2}} \frac{x^{2}}{\kappa^{-1} B^{1 / 3} m_{a}}, \\
\epsilon_{\mathrm{QCD}}^{(1)}(x)= & \frac{3}{4}\left(\frac{9 \pi}{2 g}\right)^{\frac{1}{3}} \frac{E_{B}^{1 / 4}}{x}-\frac{18 \Delta^{2}}{g E_{B}^{1 / 4}}\left(\frac{2 g}{9 \pi}\right)^{\frac{1}{3}} x \\
& +\frac{4 \pi}{3} E_{B}^{\frac{1}{4}} \theta\left(x_{1}-x\right)\left(1-\frac{x^{2}}{x_{1}^{2}}\right) x^{3} .
\end{aligned}
$$

The equilibrium point can be found following the condition

$$
0=\left.\frac{\partial \epsilon_{\mathrm{tot}}}{\partial x}\right|_{x=x_{\mathrm{eq}}} .
$$

The solution can be well approximated from numerical computation as

$$
\begin{aligned}
& \epsilon_{\mathrm{tot}}^{(1)} \simeq-0.57 m_{\pi}+\frac{3.51 m_{\pi}}{\left(\kappa^{-1} B^{1 / 3} \frac{m_{a}}{m_{\pi}}\right)^{0.310}} \\
& \text { within } \quad 0.2 \lesssim \kappa^{-1} B_{ \pm}^{1 / 3} m_{a} / m_{\pi} \lesssim 0.95
\end{aligned}
$$

This solution has accuracy up to $0.6 \%$ compared to the exact numerical solution within the range (23b), see the Appendix for more technical details. This energy per baryon charge will be used in the next section for our numerical analysis of this AQN dark matter model to test its consistency with all available constraints. 
Our second model to be considered has a very different building principle, and essentially is based on the ideas of the old constituent quark model being applied to the dense matter systems [44]. This model will serve as a complementary tool which allows us to test the sensitivity of our results to different phenomenological parameters effectively describing the strongly coupled dense QCD.

We assume the following form for the energy of the nuggets:

$$
E^{(2)}=B M_{q}+4 \pi \sigma_{\mathrm{eff}} R^{2} .
$$

Here $M_{q}$ is the effective constituent quark's mass in the CS phase in the bulk of the nugget. The $M_{q}$ should have a typical QCD scale and serves as a parameter of the model. The energy per quark degree of freedom is therefore

$$
\begin{aligned}
\epsilon_{\mathrm{tot}}^{(2)} & =M_{q}+\frac{32 \pi \chi}{\mu^{2}}\left(\frac{9 \pi}{2 g}\right)^{\frac{2}{3}} \frac{1}{\kappa^{-1} B^{1 / 3} m_{a}} \\
& \simeq M_{q}+\left(\frac{376 \mathrm{MeV}}{\mu}\right)^{2} \frac{m_{\pi}}{\kappa^{-1} B^{1 / 3} \frac{m_{a}}{m_{\pi}}},
\end{aligned}
$$

where we substitute in $\frac{R}{B^{1 / 3}}=\frac{1}{\mu}\left(\frac{9 \pi}{2 g}\right)^{\frac{1}{3}}$ from Eq. (19). We should note that $M_{q}$ and $\mu$ are not completely free parameters according to various phenomenological models for dense phases. To be consistent with previous studies (applied to the neutron star physics where this model was used) we adopt the following numerical values for the relevant parameters [44]:

$$
\left(M_{q}, \mu\right) \simeq(200,400) \mathrm{MeV}, \quad \text { and }(160,500) \mathrm{MeV} \text {. }
$$

In spite of the fact that model 2 has a fundamentally different building principle, we observed that these choices (26) for $\left(M_{q}, \mu\right)$ parameters produce results which are numerically very close to the results (23) obtained for model 1, see the Appendix for detailed analysis.

We conclude this "technical section" with the following comment. The two models discussed in this section and represented by Eqs. (23) and (25) are based on fundamentally different principles. Nevertheless, both models lead to similar results for relevant parameters, and furthermore, demonstrate that the energy density $\epsilon$ in both models depends on a single dimensionless parameter $\kappa^{-1} B^{1 / 3} m_{a} / m_{\pi}$, which is clearly a highly nontrivial feature. A more detailed discussion of these two models can be found in the Appendix.

\section{THE AQN MODEL CONFRONTING THE OBSERVATIONS}

The main purpose of this section is to analyze all possible constraints on the parameters of the AQN model.
In previous sections we introduced a number of phenomenological parameters describing this model. Our goal here is to analyze the allowed region in parametrical space where these parameters may vary but remain consistent with all known observations/experiments. We use the result (23) or (25) from Sec. IV to fit the parameters $\epsilon_{ \pm}$. We use (15) from Sec. III to fit the density of the relic dark matter propagating axions into our AQN model. Finally, we implement constraints on physically observable parameters $B, \theta_{a, i}$, $m_{a}, H_{I}$ from a large number of independent experiments and observations to analyze the allowed window for the most important parameter of the AQN model represented by the coefficient $c$. This key parameter is defined by Eq. (8) and describes the disparity between nuggets and antinuggets. As we argued in [10] this coefficient $c$ must be on the order of 1 as a result of interaction with a coherent $C P$-odd axion field. This coefficient, in principle, is calculable from first principles along with other parameters of the model as all fields, and coupling constants and interactions are represented by the standard model physics accompanied by the axion field $\theta(x)$ with a single additional fundamental parameter $f_{a}$. However, such computations presently are not feasible as even the phase diagram shown in Fig. 1 at $\theta \neq 0$ is not yet understood.

We represent our numerical results in Secs. V B and V C. However, first of all, in Sec. VA we make an overview of the known constraints on the relevant parameters of the AQN model.

\section{A. Constraints on parameters of the AQN model}

We start with Eq. (12), which defines coefficient $c$. This should not be considered as a constraint on $c$ as it essentially represents our convention that we define the visible matter as the baryons with positive baryon charge. Therefore, the absolute value of the baryon charge hidden in the antinuggets $B_{-}$must be greater than the baryon charge $B_{+}$hidden in the nuggets (as a result of global conservation of the baryon charge, which is assumed to be zero at all times). This leads to the formal relation $c<1$ which reflects our convention. The parameter $c$ is obviously a positively defined parameter which is explicitly represented by Eq. (8).

Another constraint $0.2 \lesssim \kappa^{-1} B^{1 / 3} m_{a} / m_{\pi} \lesssim 0.95$ follows from (23b). This constraint is related to our study of the stability of the nuggets in the CS phase, see Sec. IV with more technical details.

The next item we want to discuss is the known constraints on the baryon charge $B$ of the nuggets (or antinuggets) represented by (3). We have already made a few comments about the constraints related to the galactic observations, Apollo data, and ancient Mica analysis mentioned in Sec. II, footnote 3 and reviewed in $[31,32]$. Now we want to make a few comments regarding other constraints related to different observations and analysis. 
First of all we want to mention the constraints related to the limits from the total geothermal energy budget of Earth [45], which are consistent with (3). It has also been suggested that the ANITA experiment may be sensitive to the radio band thermal emission generated by these objects as they pass through the Antarctic ice [45]. These experiments may thus be capable of adding direct detection capability to the indirect evidence mentioned previously in Sec. II.

It has also been suggested recently [46] that the interactions of the antinuggets with normal matter on Earth and the Sun will lead to annihilation and an associated neutrino flux. Furthermore, it has been claimed [46] that the antiquark nuggets cannot account for more than $20 \%$ of the dark matter flux based on constraints for the neutrino flux in the $20-50 \mathrm{MeV}$ range, where sensitivity of the underground neutrino detectors such as SuperK have their highest signal-to-noise ratio. However, the claim [46] was based on the assumption that the annihilation of visible baryons with antiquark nuggets generates a neutrino spectrum similar to a conventional baryon-antibaryon annihilation spectrum when the large number of produced pions eventually decay to muons and consequently to highly energetic neutrinos in the $20-50 \mathrm{MeV}$ energy range. This claim has been dismissed in Ref. [47] by emphasizing that antinuggets cannot be treated as the usual antimatter in a conventional hadronic phase, as the quarks in nuggets (and antiquarks in antinuggets) belong to the CS phase rather than to the hadronic phase we are familiar with.

One could also expect a set of strong constraints on the antinuggets by considering the radio observations or strong $511 \mathrm{keV}$ line emission from nearby galaxies if one assumes that the antinuggets (almost) saturate the corresponding emissions in our Milky Way. Indeed, similar consideration in other models essentially rules out the dark matter explanation of the $511 \mathrm{keV}$ line observed in our Galaxy. However, the corresponding analysis carried out in [48] for the radio radiation and in [49] for $511 \mathrm{keV}$ line emission from nearby galaxies does not lead to any new constraints, in huge contrast to conventional WIMP models, which typically predict radiation from nearby galaxies exceeding the observed values. We refer to the original papers $[48,49]$ for details and references.

Our last comment related to constraints on $B$ as given by (3) is related to the recent arguments [33] advocating that the EUV and soft $\mathrm{x}$-ray emission from the solar corona might be due to the antiquark nuggets hitting the Sun from outer space. If one identifies the observed nanoflares with annihilation events of the antinuggets in the solar corona one can infer the nuggets' baryon charge distribution from the (measured) energy distribution of the nanoflares in the solar corona, see also some related comments in Sec. VI. This identification leads to an independent estimate [33] on possible values for $B$ which is perfectly consistent with the previous studies, which we express as

$$
10^{23} \lesssim B \lesssim 10^{28}
$$

In the numerical analysis which follows we treat the window (27) as the solid constraint of the allowed magnitude of the nuggets' baryon charge.

We next consider the classical window ${ }^{9}$ for axion mass, see the recent review paper [21]:

$$
10^{-6} \mathrm{eV} \lesssim m_{a} \lesssim 10^{-2} \mathrm{eV}
$$

By using the relation (17) this window for $m_{a}$ can be expressed in terms of the corresponding classical window for $f_{a}$ :

$$
5.7 \times 10^{8} \mathrm{GeV} \lesssim f_{a} \lesssim 5.7 \times 10^{12} \mathrm{GeV}
$$

One should emphasize that the constraint (28) or equivalently (29) is the commonly accepted axion window, and it does not, by any means, originate from our analysis of the AQN model. Nevertheless, all our constraints depend on $m_{a}$ as it explicitly enters the Eq. (23b).

From these discussions it is clear that the axion mass $m_{a}$ plays a dual role in our analysis because it enters the formulas related to the physics of the nuggets as Eq. (23b) states. It also enters the expression (15) for $\Omega_{a}$. It unambiguously implies that the remaining portion of the dark matter represented by the nuggets' contribution (13) becomes also (implicitly) highly sensitive to $m_{a}$ through dependence of the axion portion of the dark matter represented by $\Omega_{a}$.

As we argue below, for reasonable values of $\kappa$ in the range $10^{-4} \lesssim \kappa \lesssim 10^{-2}$, the constraints (23b), (27), and (28) become mutually compatible, which we consider a highly nontrivial consistency check as all the parameters entering these relations have been constrained by very different physics related to independent observations, experiments, and analysis.

The next constraint to consider is related to analysis of the inflationary scale $H_{I}$ and the related constraints on the tensor-to-scalar ratio and the isocurvature perturbations. The basic assumption of this work is that PQ symmetry breaking occurs before/during inflation, in which case

$$
f_{a}>H_{I} / 2 \pi
$$

\footnotetext{
${ }^{9}$ For the main purposes of this paper, we will only consider the "classical axion window," where the initial misalignment angle $\theta_{a, i}$ is not fine-tuned. Note that while the upper bound is a very solid constraint as it is given by stellar physics (e.g. see review [17]), the lower bound on the axion mass in Eq. (28) should be treated as an order of magnitude estimate provided that $\theta_{a, i}$ is not fine-tuned. If the fine-tuning is allowed, $\theta_{a, i}$ may assume an arbitrarily small value, in which case the corresponding lower bound on $m_{a}$ is shifted. The only exclusion interval in this case $6 \times 10^{-13} \mathrm{eV}<m_{a}<2 \times 10^{-11} \mathrm{eV}$ is obtained from black hole superradiance effects [50].
} 
see e.g. [20] for review. This assumption plays a crucial role in our analysis $[9,10]$ because the $C P$-odd axion field must be coherent on the enormous scale of the entire Universe to separate the baryon charges on these gigantic scales with the same sign of $\theta$. Precisely this coherent axion field generates the disparity between the nuggets and antinuggets which eventually leads to the generic and fundamental prediction (1) of this entire framework.

It is known that the inflationary Hubble scale is tied to the value of the tensor-to-scalar ratio $r_{T}$ which measures $H_{I}$. Assuming the simplest single field inflationary model, the nonobservation of the tensor modes $\left(r_{T}<0.12\right)$ imposes the upper limit for the inflation scale, see [20,51]:

$$
H_{I} / 2 \pi \lesssim 1.4 \times 10^{13} \mathrm{GeV}
$$

An important comment here is that (31) is a highly modeldependent result and varies from one inflationary model to another. It is presented here exclusively for illustrative purposes to provide some orientation for the relevant scales of the problem.

The isocurvature perturbations related to the axion field provide another independent constraint on $H_{I}$. We recall that the amplitude for the isocurvature power spectrum is determined by the following expression, see the original papers [52-56] and review article [20]:

$$
A_{I}=\left(\frac{\Omega_{a}}{\Omega_{\mathrm{DM}}}\right)^{2} \cdot\left(\frac{H_{I}}{\pi \phi_{i}}\right)^{2}, \quad \phi_{i} \equiv f_{a} \theta_{a, i} .
$$

The corresponding isocurvature amplitude is strongly constrained by a CMB analysis, $A_{I} / A_{s}<0.038$, where $A_{s}$ is the conventional amplitude for the scalar power. It is normally assumed that the nonobservation of the isocurvature perturbation provides a strong constraint on axion properties in a scenario where the PQ symmetry is broken before/during inflation. Our original comment here is that the axion contribution represented by $\Omega_{a}$ in Eq. (32) to the dark matter density $\Omega_{\mathrm{DM}}$ could be numerically quite small in the AQN model as the nuggets in most cases play the dominant role by saturating the dark matter density. Such a scenario drastically alleviates some severe constraints on parameters in a conventional analysis, where one normally assumes that the axions saturate the dark matter density.

We conclude this subsection with the following remark. The conventional analysis on the relation between dark matter axions, inflationary scale, and isocurvature perturbations very often assumes that the axions saturate the dark matter density. It should be contrasted with our AQN model where the axions themselves with the same $f_{a}$ may contribute very little to $\Omega_{\mathrm{DM}}$ as the dominant contribution may come from the nuggets, which always satisfy the relation $\Omega_{\mathrm{DM}} \sim \Omega_{\mathrm{visible}}$ according to (1) irrespective of $f_{a}$ or the initial misalignment angle $\theta_{a, i}$. It may alleviate some severe constraints on the parameters (such as $H_{I}, f_{a}, r_{T}$, $\left.\theta_{a, i}\right)$ which other models normally face.

\section{B. Numerical plots}

The goal of this subsection is to analyze the dependence of the internal (with respect to the AQN model) parameter $c$ from external parameters of the system such as $B, \theta_{a, i}, m_{a}$, $H_{I}$ which are well-defined observables irrespective of the specific features of the AQN model. As the parameter $c$ cannot be negative or larger than 1 , the corresponding plots provide us with information on the typical values of the external parameters $B, \theta_{a, i}, m_{a}, H_{I}$ when the AQN model is self-consistent with all presently available constraints.

We start our analysis by plotting in Fig. 2(a) the parameter $c$ as a function of $m_{a}$ and $B$, where we fix specific values for parameters $\kappa=10^{-4}$ and $H_{I} / 2 \pi=5.7 \times$ $10^{8} \mathrm{GeV}$ and $\theta_{a, i}=10^{-3}$ to simplify the arguments and analysis. We also plotted the $f_{a}=\frac{H_{I}}{2 \pi}$ with a red solid line to localize the physical parametric space and remove unphysical (within the AQN model) solutions. We also plotted (by green and blue dashed lines) the region in parametrical space where the condition (23b) is satisfied and our computations in the CS phase are justified. For this specific choice of parameters one can explicitly see that parameter $c$ is constrained in a parallelogram with the range $0.4 \lesssim c \lesssim 0.6$. This region of the parametrical space satisfies all internal and external constraints listed in the previous section.

From the same plot one can also identify the allowed region of the nuggets' baryon charge $B$ for a given axion mass $m_{a}$. One should emphasize that the dark matter density in Fig. 2(a) for $\theta_{a, i}=10^{-3}$ is entire saturated by the nuggets, as the direct axion production is strongly suppressed by the small initial misalignment angle $\theta_{a, i}=10^{-3}$. To see the role of the direct axion production one can choose $\theta_{a, i}=10^{0}$, as shown in Fig. 2(b). In this case the direct axion production saturates the dark matter density $\Omega_{\mathrm{DM}}=\Omega_{a}$ at small axion masses $m_{a} \simeq 10^{-5} \mathrm{eV}$, as shown by the solid yellow line. When $10^{-3}<\theta_{a, i}<1$ varies between these two values the corresponding allowed region for the nuggets' parametrical space $\left(B, m_{a}\right)$ will be modified accordingly as the allowed region for the nuggets obviously shrinks when the direct axion production starts to play an essential role.

The key observation here is that there will always be a region $\left(B, m_{a}\right)$ when the total dark matter density assumes its observational value through the parameter $c$ which determines the nuggets' contribution to $\Omega_{\mathrm{DM}}$. The corresponding contribution varies to accommodate the related axion portion $\Omega_{a}$ as the total dark matter density $\Omega_{\mathrm{DM}}$ in Fig. 2 is fixed and assumes its observational value.

In Fig. 3 we wish to demonstrate the sensitivity of the allowed region for region $\left(B, m_{a}\right)$ with respect to the $H_{I} / 2 \pi$ parameter, where the constraint (31) is applied. 


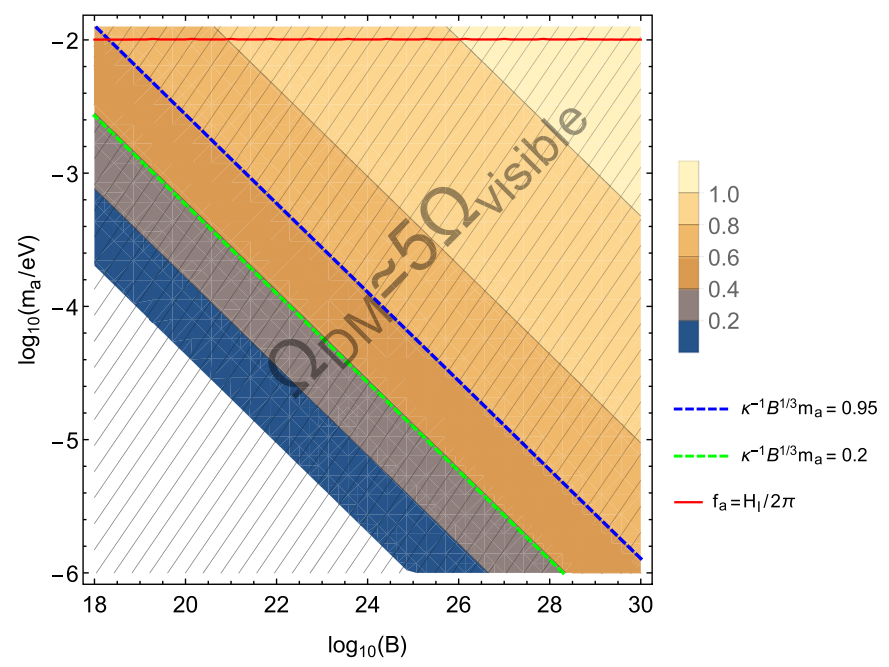

(a) $H_{I} / 2 \pi=5.7 \times 10^{8} \mathrm{GeV}, \theta_{a, i}=10^{-3}$

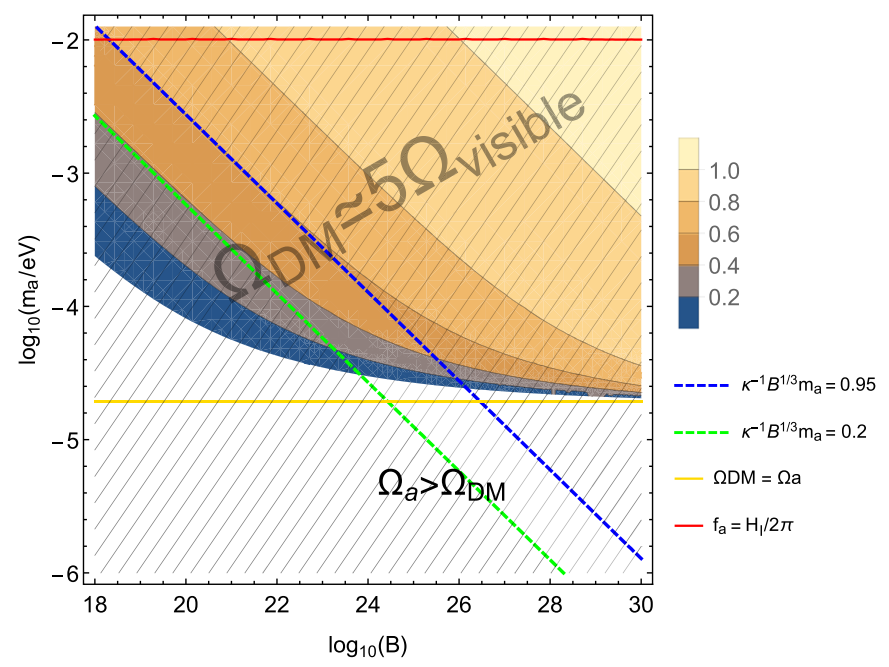

(b) $H_{I} / 2 \pi=5.7 \times 10^{8} \mathrm{GeV}, \theta_{a, i}=10^{0}$

FIG. 2. Contour plots of $c$ as a function of $m_{a}$ and $B$ for (a) $\theta_{a, i}=10^{-3}$ and (b) $\theta_{a, i}=10^{0}$, respectively, with the fixed values $H_{I} / 2 \pi=5.7 \times 10^{8} \mathrm{GeV}$ and $\kappa=10^{-4}$. Different colors (shown on the right) correspond to the different values of the parameter $c \sim 1$. Precisely this parameter $c$ essentially determines the relation between dark and visible matter according to approximate relation (4). Here $B$ corresponds to the baryon number of an antinugget (which is also the case for other figures in this subsection).

We want to demonstrate the corresponding sensitivity to the $H_{I} / 2 \pi$ parameter by showing that there is not any dependence on $H_{I}$ for a sufficiently small $H_{I} / 2 \pi \simeq$ $10^{10} \mathrm{GeV}$, as shown in Fig. 3(a). In all respects the plot is very much the same as the one shown in Fig. 2(a). In both cases the dark matter density is dominated by the nuggets, and the allowed region $\left(B, m_{a}\right)$ is not sensitive to the $H_{I} / 2 \pi$ value as long as the Hubble parameter is sufficiently small. However, when $H_{I} / 2 \pi$ becomes close to $f_{a} \sim 10^{11} \mathrm{GeV}$, the window for $c$ is shifted as shown in Fig. 3(b) to accommodate the conventional contribution of the propagating axions. The main point is that there will always be a region $\left(B, m_{a}\right)$ when the total dark matter density assumes its observational value, though the magnitude of $c \in(0,1)$ assumes somewhat different values, depending on the external parameters of the system.

Our next task is to analyze the sensitivity of our results to the QCD parameters related to the CS properties of the nuggets. To accomplish this goal we plot parameter $c$ in Fig. 4 as a function of $m_{a}$ and $B$ using $\epsilon_{\text {tot }}^{(2)}$ for model 2 determined by Eqs. (25) and (26). The corresponding plot for $\left(M_{q}, \mu\right)=(200,400) \mathrm{MeV}$ is presented in Fig. 4(a), while the plot for $(160,500) \mathrm{MeV}$ is shown in Fig. 4(b).

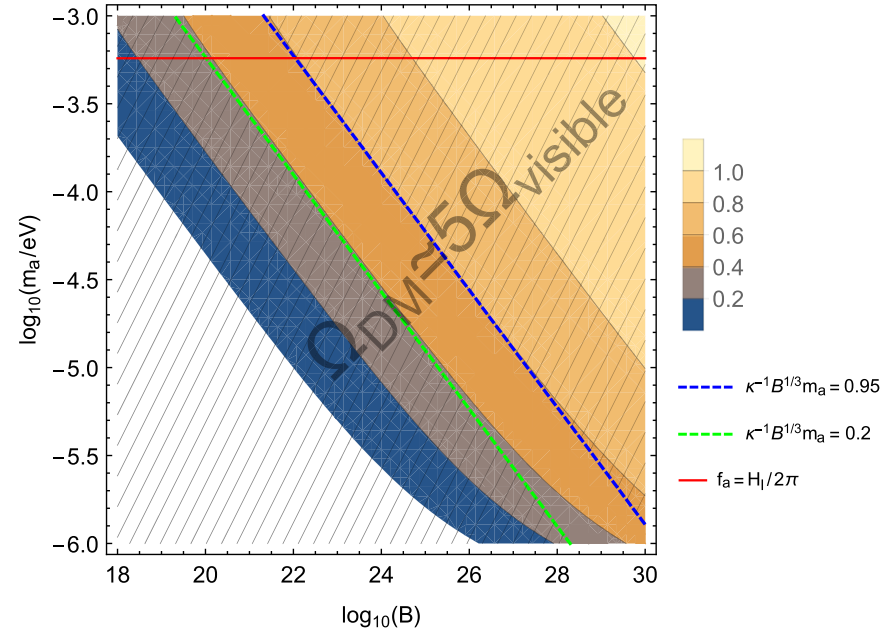

(a) $H_{I} / 2 \pi=10^{10} \mathrm{GeV}, \theta_{a, i}=10^{-1}$

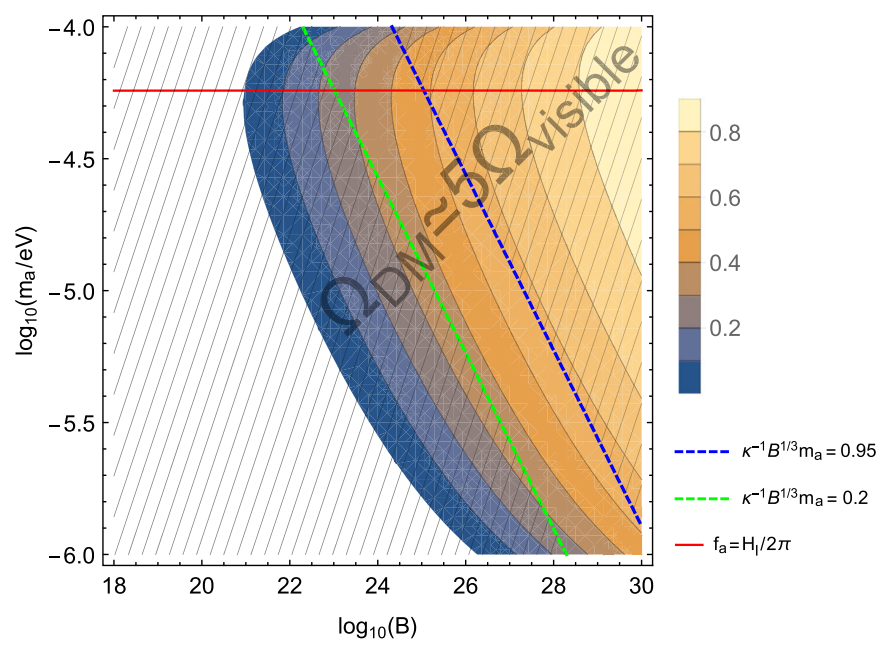

(b) $H_{I} / 2 \pi=10^{11} \mathrm{GeV}, \theta_{a, i}=10^{-1}$

FIG. 3. Contour plots of $c$ as a function of $m_{a}$ and $B$ for (a) $H_{I} / 2 \pi=10^{10} \mathrm{GeV}$ and (b) $H_{I} / 2 \pi=10^{11} \mathrm{GeV}$, respectively, with the fixed value $\theta_{a, i}=10^{-1}$ and $\kappa=10^{-4}$. Different colors (shown on the right) correspond to the different values of the parameter $c \sim 1$. 


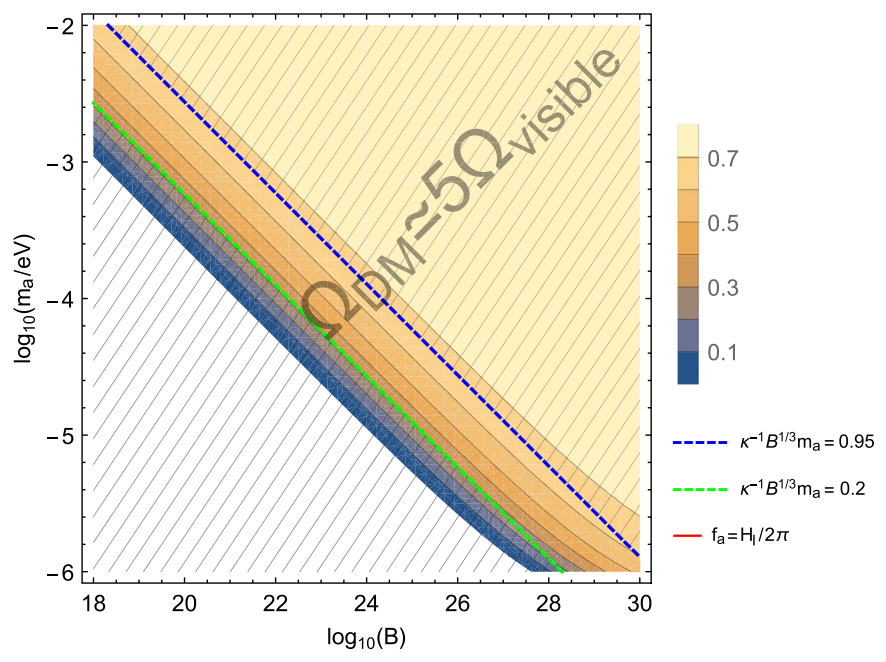

(a) $H_{I} / 2 \pi=5.7 \times 10^{8} \mathrm{GeV}, \theta_{a, i}=10^{-1}$

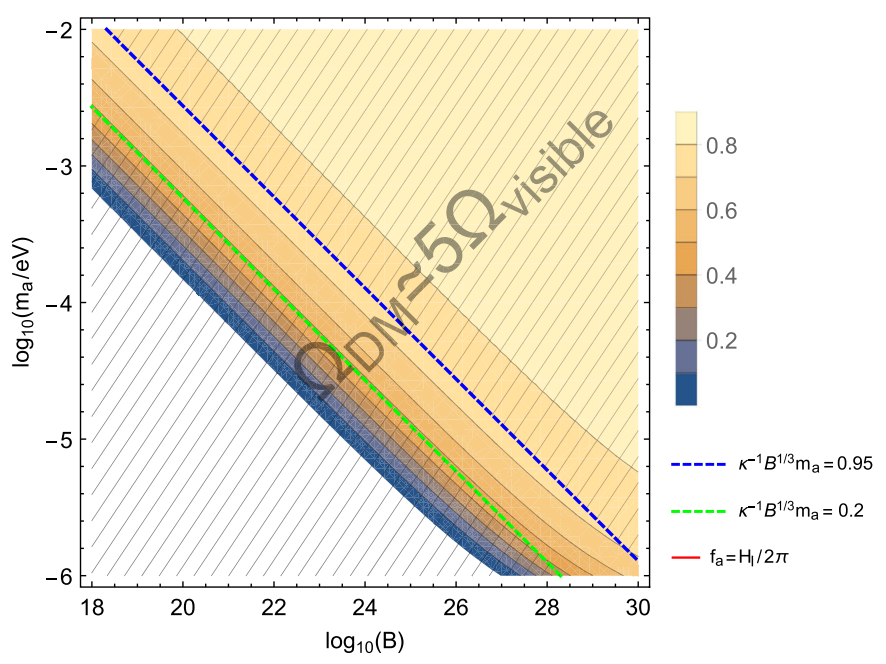

(b) $H_{I} / 2 \pi=5.7 \times 10^{8} \mathrm{GeV}, \theta_{a, i}=10^{-1}$

FIG. 4. Model 2. Contour plots of $c$ as a function of $m_{a}$ and $B$ for specific values of $H_{I}, \theta_{a, i}$, and $\kappa=10^{-4}$. (a) $\left(M_{q}, \mu\right)=(200,400) \mathrm{MeV}$. (b) $\left(M_{q}, \mu\right)=(160,500) \mathrm{MeV}$. Different colors (shown on the right) correspond to the different values of the parameter $c \sim 1$.

The main conclusion is that model 2 (which is based on fundamentally different building principles than model 1) with various parameters produces nevertheless the quantitatively similar results as model 1 analyzed previously and shown in Fig. 2(a). A more detailed comparison presented in the Appendix supports this claim. This conclusion essentially implies that our phenomenological results are not very sensitive to the specifics of the QCD parametrization of the system describing the dense CS phase of matter in a strongly coupled regime. Therefore, we treat our results as the solid consequences of the AQN model.

As an additional note, the parameter $c_{\Omega}$ as defined by Eqs. (7) and (9) and which describes the mass difference between the nuggets and antinuggets (in contrast to parameter $c$, which describes the baryon charge difference between the nuggets and antinuggets) is numerically very close to the parameter $c$ studied above. Specifically, one can show that for model 1 (23) the parameter $c_{\Omega} \simeq 1.17 c$ is within $15 \%$ accuracy for the region $0.4 \lesssim c \lesssim 0.6$ which dominates the parametrically allowed region, as discussed in the preceding paragraphs. Therefore, we do not show the plots for $c_{\Omega}$ as a function of external parameters because they are very similar to the plots for $c$ presented and discussed above.

\section{No fine-tuning in the AQN scenario}

As we mentioned in Sec. III, the dark matter propagating axion itself may not saturate the total dark matter. In contrast, the nuggets' formation always generates a large contribution $\Omega_{\mathrm{DM}} \sim \Omega_{\text {visible }}$ and always accompanies the conventional axion production. This property of the AQN model is demonstrated in Fig. 5, where we plot $\Omega_{a} / \Omega_{\mathrm{DM}}$ as a function of $m_{a}$ and $\phi=\sqrt{\theta_{a, i}^{2}+\left(H_{I} /\left(2 \pi f_{a}\right)\right)^{2}}$. The function $\phi\left(\theta_{a, i}, H_{I}\right)$ enters formula (15) for $\Omega_{a}$ and counts together with the initial homogeneous displacement contribution and the backreaction contribution to the free dark matter axions. Figure 5 explicitly shows that $m_{a}$ and $\phi$ have to be highly fine-tuned to make $\Omega_{a}$ saturate $\Omega_{\mathrm{DM}}$ exactly, shown as a bright green solid line. In other words, for a specific magnitude of $m_{a}$ there is a single value of $\phi$ when the dark matter density assumes its observable value. Once these two parameters, $m_{a}$ and $\phi$, slightly deviate from the appropriate values, $\Omega_{a}$ strongly deviates from $\Omega_{\mathrm{DM}}$.

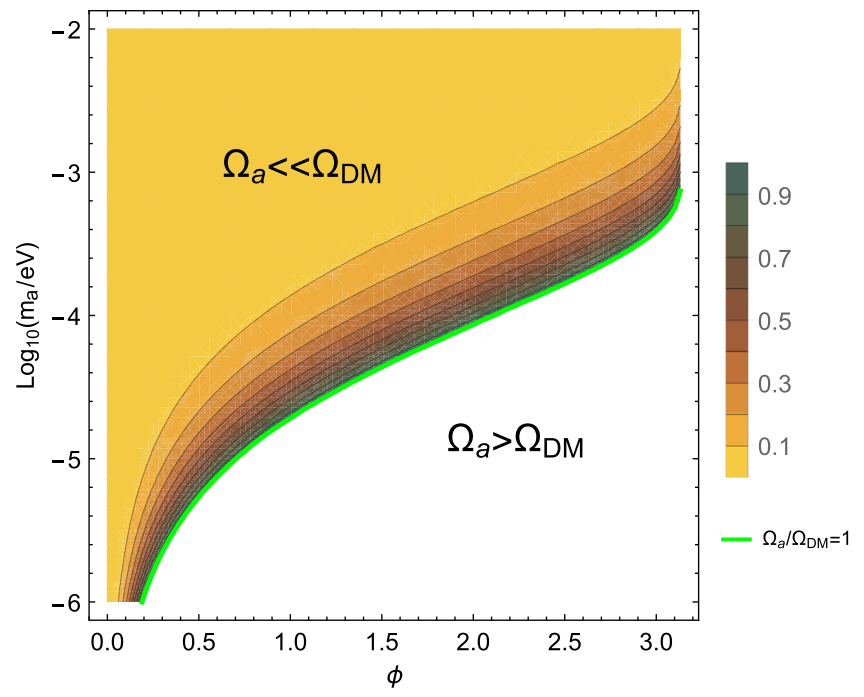

FIG. 5. Contour plots of $\Omega_{a} / \Omega_{\mathrm{DM}}$ as a function of $m_{a}$ and $\phi=\sqrt{\theta_{a, i}^{2}+\left(H_{I} /\left(2 \pi f_{a}\right)\right)^{2}}$. Different colors (shown on the left) correspond to the different values of $\Omega_{a} / \Omega_{\mathrm{DM}}$. 
This conventional fine-tuning scenario should be contrasted with the results of the AQN model when $\Omega_{a}$ may contribute very little to $\Omega_{\mathrm{DM}}$. Nevertheless, the $\Omega_{\mathrm{DM}}$ assumes its observation value as a result of an additional nugget's contribution which always accompanies the axion production and always generates a contribution of order 1 , as we have already emphasized. In other words, the AQNs play the role of the "remaining" DM density which, in fact, could be the dominating portion of the $\Omega_{\mathrm{DM}}$. As we have seen in Sec. V B, there will always be a contribution to the DM model constituted of free axions and the AQNs for the allowed parametric space. In other words, for a specific magnitude of $m_{a}$ there is a large window of $\phi$ corresponding to the different values of parameter $c \in(0,1)$ when the dark matter density assumes its observable value. Different colors in Fig. 5 correspond to the different axion contribution represented by parameter $\Omega_{a} / \Omega_{\mathrm{DM}}$. Therefore, the fine-tuning problem does not even occur in the AQN scenario, as the entire parametrical space on the left from the green solid curve $\Omega_{a}=\Omega_{\mathrm{DM}}$ corresponds to the observable dark matter density. The white region in Fig. 5 is excluded because the requirement $\Omega_{a} \leq \Omega_{\mathrm{DM}}$ is violated.

As the final technical remark we also notice from Fig. 5 that for most of the parameter space, we have $\Omega_{a} \lesssim 0.2 \Omega_{\mathrm{DM}}$. To make the above statement more precise, we plot in Fig. 6 the ratio $\Omega_{a} / \Omega_{\mathrm{DM}}$ as a function of $c$ and parameter $\kappa^{-1} B^{1 / 3} m_{a} / m_{\pi}$ determined by the QCD physics, as given by Eq. (23). The white region in Fig. 6 stands for the excluded region of parameters $\left(c, \kappa^{-1} B^{1 / 3} m_{a}\right)$. This plot shows that the parameter $c$ cannot be very close to $\sim 1$ for the allowed QCD window $0.2 \lesssim \kappa^{-1} B^{1 / 3} m_{a} / m_{\pi} \lesssim 0.95$. This property, in fact, can be understood analytically from Eq. (4) or its generalized version Eq. (14) where $c \rightarrow 1$ implies $\Omega_{\mathrm{DM}} \gg \Omega_{\text {visible, }}$, which violates the observable

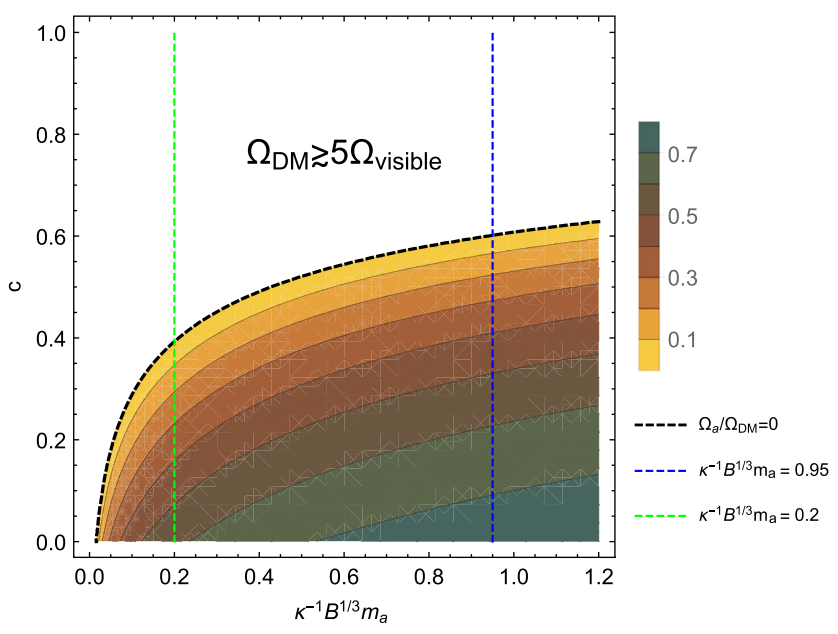

FIG. 6. Contour plots of $\Omega_{a} / \Omega_{\mathrm{DM}}$ as a function of $c$ and $\kappa^{-1} B^{1 / 3} m_{a}$. Different colors (shown on the right) correspond to the different values of $\Omega_{a} / \Omega_{\mathrm{DM}}$. relation $\Omega_{\mathrm{DM}} \simeq 5 \Omega_{\text {visible }}$. The main conclusion to be drawn from this plot is that the parametrical region where $\Omega_{\mathrm{DM}}$ assumes its observable value is very large and perfectly consistent with the QCD constraints related to parameters $c$ and $\kappa^{-1} B^{1 / 3} m_{a} / m_{\pi}$.

This conclusion is another manifestation of the basic consequence of the AQN framework when the relation (1) is not sensitive to any details of the system, such as $m_{a}$ or $\theta_{a, i}$, but rather represents a direct outcome of this proposal. This fundamental result is essentially incorporated into the initial building principle of the entire framework, and cannot disappear as a result of some additional technical details and modifications.

\section{CONCLUSION AND FUTURE DEVELOPMENT}

This work is the first attempt to extract the key element of the proposal, the coefficient $c$ describing the disparity between nuggets and antinuggets, as defined by Eq. (4) or its generalized version Eq. (14), from the observational constraints in the quantitative way. Precisely this asymmetry eventually determines the dark matter density (within this framework) we observe today as a result of the charge separation mechanism replacing the conventional baryogenesis scenario as discussed in the overview in Sec. I. This work, to a large extent, is motivated by the recent progress in the axion search experiments which, hopefully, in a few years should cover almost the entire interesting region of the allowed parametrical space for $m_{a}$ as recently reviewed in [19-22].

A distinct feature of the AQN model is that the fundamental relation (1) is always satisfied in this framework irrespective of the parameters of the system, such as the axion mass $m_{a}$ or misalignment angle $\theta_{a, i}$. Therefore, the discovery of the axion with mass $m_{a}$ which gives only a fraction of the observed dark matter density $\Omega_{a}<\Omega_{\mathrm{DM}}$ is perfectly consistent with our scenario as the remaining portion of the dark matter is generated by the nuggets' contribution (6), which always accompanies the axion production within this proposal.

The corresponding numerical results are presented in Sec. VB and explicitly demonstrate that this model is perfectly consistent with the various constraints listed in Sec. VA. One should emphasize that the corresponding constraints have been derived from a variety of astrophysical, cosmological, satellite, and ground based observations. Furthermore, there are a number of frequency bands where some excess of emission was observed, but not explained by conventional astrophysical sources. This model may explain some portion, or even the entire excess of observed radiation in these frequency bands, see the short review [31]. This model is also consistent with known constraints from the axion search experiments. Finally, in Sec. V C we argued that the corresponding results are not very sensitive to various QCD parameters describing the dense CS phase of the AQNs. 
Therefore, there is not much sense in repeating here, in conclusion, the main results of Secs. VA, V B, and V C. Rather, we would like to discuss the implications of these results on future related studies.

In particular, it has been recently argued that the dark matter axions can generate peculiar effects in the so-called Josephson junctions [57-59]. If a small measured peak of unknown origin is identified with the dark matter axions as suggested in [57-59], one can infer that the axion mass should be

$$
m_{a}=(104.0 \pm 1.3) \times 10^{-6} \mathrm{eV}, \quad \rho_{a} \simeq 0.1 \frac{\mathrm{GeV}}{\mathrm{cm}^{3}},
$$

where we also quote the estimated axion dark matter density $\rho_{a}$, which was required to interpret the measured signal in the Josephson junctions as a result of the DM axion [59]. This value of $\rho_{a}$ represents approximately a quarter of the expected dark matter density in the halo. One should emphasize that the estimate (33) for the axion mass was extracted from an analysis of four different experiments observed by four different groups pointing to the same mass value (33). Therefore, it is very unlikely that (33) is a statistical fluke.

If one literally accepts the measured signal in the Josephson junctions as a result of the DM axion (33) one could immediately infer, for example, from Fig. 5 that for $m_{a} \simeq 10^{-4} \mathrm{eV}$ and $\Omega_{a} / \Omega_{\mathrm{DM}} \simeq 0.3$, as Eq. (33) would suggest, the misalignment angle $\theta_{a, i}$ should be on the order of 1 provided that $\left(H_{I} / 2 \pi f_{a}\right) \ll 1$. Furthermore, from Fig. 2(b) for the axion mass $m_{a} \simeq 10^{-4} \mathrm{eV}$ and $\theta_{a, i} \simeq 1$ one could estimate the average baryon charge of the AQNs, which is estimated to be on the order of $B \sim 10^{25}$. This estimate is very encouraging as it is perfectly consistent with our previous phenomenological analysis reviewed in Sec. II.

One should emphasize that such estimates and selfconsistency checks are very important for subsequent development and future studies. For example, in Ref. [33] it has been argued that the Sun could serve as an ideal lab to study the AQN model. The main point is that the antinuggets deposit a huge amount of energy in the corona as a result of annihilation events with the solar material. It may explain the so-called the Solar Corona Mystery, where the temperature of the corona is about $10^{6} \mathrm{~K}$, i.e., a few hundred times hotter than the solar surface temperature. It may also explain the extreme UV and soft $\mathrm{x}$-ray emissions from the corona, which is very hard to explain using conventional astrophysical processes. It has been also suggested in Ref. [33] that the observed brighteninglike events called nanoflares in the Sun can be identified with the annihilation events of the antinuggets, in which case the observed energy distribution of the nanoflares must coincide with the baryon charge distribution studied in the present work. In other words, the energy distribution of the nanoflares and the baryon charge distribution of the nuggets are one and the same function within the AQN model [33].

This statement can be formally expressed as follows:

$$
d N \sim B^{-\alpha} d B \sim W^{-\alpha} d W,
$$

where $d N$ is the number of nanoflare events per unit time with energy between $W$ and $W+d W$ which occur as a result of complete annihilation of the antinuggets carrying the baryon charges between $B$ and $B+d B$. These two distributions are tightly linked as these two entities are related to the same AQN objects when the annihilation of the antinuggets' baryon charge generates the energy which is interpreted as the observed nanoflare event within AQN model. The modeling and observations of the nanoflares in the corona suggest $\alpha \sim 2$, see [33] for details and references. One should emphasize that this interpretation is consistent with constraints on $B$, see (3), Refs. [31,32] for reviews, and footnote 3 for some additional remarks. Therefore, further studies of the nanoflares in the solar corona may shed some light on the nature of dark matter.

In fact this interpretation may receive further support through a future analysis of some specific correlations studied in [60]. If a subsequent development along the lines advocated for in [60] suggests that the frequency of the flares observed in the sun is directly related to dark matter particles (called "invisible matter" in Ref. [60]), it would be a major breakthrough not only in our understanding of the solar corona, but also in our understanding of the nature of dark matter.

The first theoretical estimates providing a specific mechanism on how the correlations observed in [60] could be, in principle, explained were recently suggested in [34]. The basic idea of [34] is that the nuggets entering the solar corona will inevitably generate the shock waves, as the typical velocities of the nuggets are much higher that the speed of sound in the solar atmosphere. The shock waves due to the AQNs may serve as the triggers igniting the large flares which are shown to be correlated with the invisible matter of Ref. [60]. This mechanism would also relate naively unrelated entities such as the axion, its mass, the baryon charge distribution $B$ of the nuggets (34), the flares' intensity, and their frequency of appearance on the Sun.

Our next comment about possible future studies is related to recent activities of the axion search experiments. We want to specifically mention some present and future axion search experiments such as ADMX and ADMX-HF [61], IAXO [62], CAST [63], ORPHEUS [64], MADMAX [65], and ORGAN [66]. These, and possibly many other experiments, should eventually discover the QCD axion irrespective of the assumption on validity of the axionic Josephson effect leading to (33). The only original comment we would like to make is that the discovery of the axion with mass $m_{a}$, which may generate only a small fraction of the observed dark matter density $\Omega_{a} \ll \Omega_{\mathrm{DM}}$, 
nevertheless would be a major discovery because the remaining portion of the dark matter could be generated by the nuggets' contribution (6), which always accompanies the conventional axion production within the AQN scenario, and always satisfies the generic relation (1). This would conclude a long and fascinating journey of searches for this unique and amazing particle conjectured almost 40 years ago.

We want to make one more comment on the axion search experiments when the observable is sensitive to the axion amplitude $\theta$ itself, in contrast to conventional proposals, which are normally sensitive to the derivatives $\sim \partial_{\mu} \theta$. The basic idea of the proposal [67] is that $\theta$ becomes a physically observable parameter even in the Abelian Maxwell QED if the system is defined in a topologically nontrivial sector. This can be easily achieved by placing the system into the background of an external magnetic field. The phenomenon in all respects is very similar to the wellknown Witten effect when the $\theta$ parameter becomes a physical observable in the presence of the magnetic monopole. This novel phenomena was coined as the topological Casimir effect, and there are some specific ideas on how to design and fabricate the corresponding apparatus (the so-called aKWISP project), see the talk by Cantatore [68] for relevant information.

One more possible direction for future studies from our "wish list" is a development of the QCD-based technique related to nugget evolution, cooling rates, evaporation rates, viscosity, transmission/reflection coefficients, etc., in an unfriendly environment with nonvanishing $T, \mu, \theta$ values. The problem here is that the axion mass scale $m_{a}$ and the Hubble scale $H(T)$ at the QCD epoch are drastically different from the QCD scale itself $\left(\sim \Lambda_{\mathrm{QCD}}\right)$ describing the CS phase and the nuggets' structure. In similar circumstances some researches normally change the scales (in which case the relevant parameters obviously assume some unphysical values) to attack the problem numerically. After the numerical computations are done, one can return to the physically relevant values for the parameters by using plausible arguments with some specific assumptions (which may or may not be correct) on the scaling features of the parameters when they assume their physical values.

Our goal in the near future is to develop some numerical methods and approaches to study the real time evolution of nuggets with real physical parameters which assume drastically different scales. If the project turns out to be successful it would be a major technical step forward relating to the analysis of Refs. $[9,10]$, which was devoted to the formation period of the nuggets at high temperature, and the present work, which deals with the present epoch of the cold Universe, a billion years after the nuggets had been formed.

\section{ACKNOWLEDGMENTS}

This work was supported in part by the National Science and Engineering Research Council of Canada.

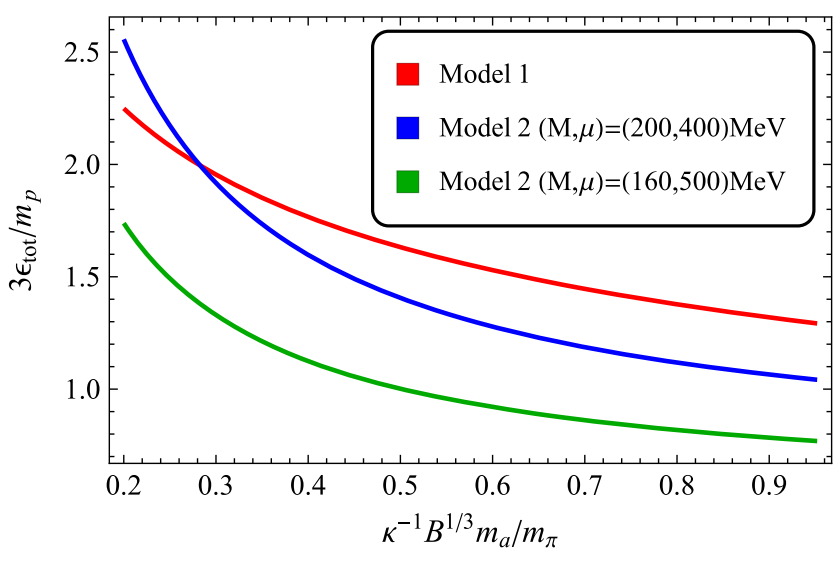

FIG. 7. Plot of $3 \epsilon_{\mathrm{tot}} / m_{p}$ vs $\kappa^{-1} B^{1 / 3} m_{a} / m_{\pi}$.

\section{APPENDIX: COMPARISON OF THE MODELS}

Our goal here is to give an overview of the different QCD-inspired models which we used in our estimates for the energy per baryon charge in the CS phase in Sec. IV. First, we note that both models, model 1 [Eq. (23)] and model 2 [Eq. (25)] are sensitive to a single dimensionless parameter $\kappa^{-1} B^{1 / 3} m_{a} / m_{\pi}$. In fact, the two models give similar curves of total energy per baryon charge (which includes the energy related to the axion domain wall $\epsilon_{\mathrm{DW}}$ as well as the Fermi energy of the quarks $\epsilon_{\mathrm{QCD}}$ ). It is quite a remarkable result, as the models are based on fundamentally different building principles.

The results are shown in Fig. 7, where we have rescaled it by hadronic energy density $\frac{1}{3} m_{p}$ per baryon charge. The short conclusion is as follows. Although the two models are built from fundamentally different principles, they turn out to give similar results. Such close agreement supports the estimations of the energy density of a stabilized quark nugget we used in Sec. IV.

In addition to this main conclusion we want to make some additional comments. In the plot shown in Fig. 7 we do not extend the analysis beyond the region when parameter $\kappa^{-1} B^{1 / 3} m_{a} / m_{\pi}$ becomes sufficiently large. The main reason to limit ourselves by this window is that the larger values of the parameter $\kappa^{-1} B^{1 / 3} m_{a} / m_{\pi}>0.95$ correspond to a smaller chemical potential. ${ }^{10}$ For the same reason, only plots in the region $\kappa^{-1} B^{1 / 3} m_{a} / m_{\pi} \leq 0.95$ have been considered in this work.

\footnotetext{
${ }^{10}$ More specifically, $\mu$ can be evaluated from Eq. (20), where $x_{\mathrm{eq}}$ is numerically solved as a function of $\kappa^{-1} B^{1 / 3} m_{a} / m_{\pi}$ from condition (22). The large value of $\kappa^{-1} B^{1 / 3} m_{a} / m_{\pi}>0.95$ corresponds to the small chemical potential $\mu \lesssim 330 \mathrm{MeV}$. At this relatively small value of $\mu$, our treatment of the dense matter as the color superconductor is not justified. The reason is that $\mu \lesssim$ $330 \mathrm{MeV}$ corresponds to conventional stable nuclear matter, which should be treated differently as the relevant degrees of freedom are hadrons, rather than quarks.
} 


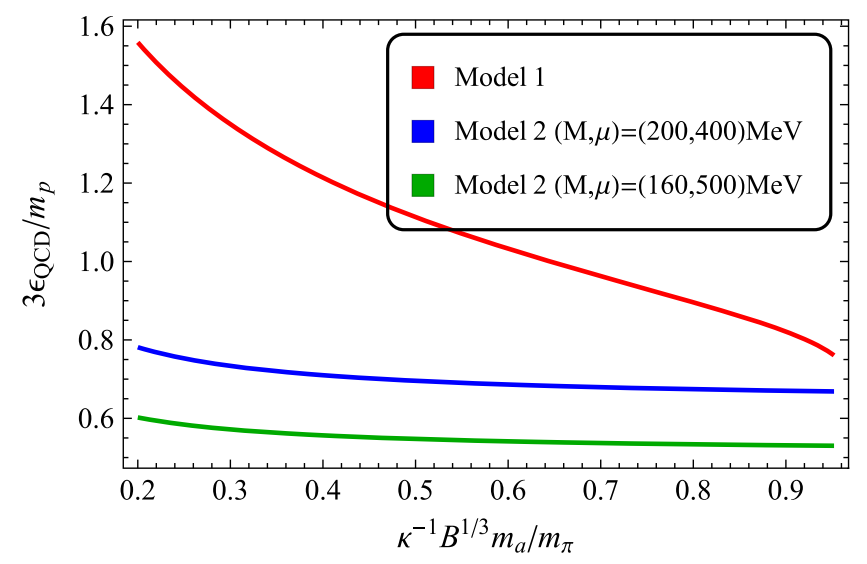

FIG. 8. Plot of $3 \epsilon_{\mathrm{QCD}} / m_{p}$ vs $\kappa^{-1} B^{1 / 3} m_{a} / m_{\pi}$.

This constraint does not imply that very large nuggets cannot exist. In fact the quark nuggets can perfectly coexist with conventional nuclear matter making an absolutely stable object, when the hadronic nuclear matter is surrounding the dense quark nugget. Such objects could be captured by stars or planets and stay in the cores of the astronomical object indefinitely. ${ }^{11}$ They can also accrete the visible hadronic matter during a long Hubble evolution since the formation time at $T \simeq 41 \mathrm{MeV}$, as shown in Fig. 1 . However, an antinugget made of antimatter will annihilate its baryon charges when it is in contact with visible matter. As the main phenomenological manifestation of the AQN model is precisely such annihilation events of the antinuggets with visible matter, in the present work we present our plots for the antinuggets and their baryon charges $B$.

The absolute stability of the AQNs is determined by the condition $\frac{3 \epsilon_{\mathrm{QCD}}}{m_{p}} \leq 1$, while the metastability (with a very long lifetime exceeding the lifetime of the Universe) of this condition may not be strictly satisfied. The corresponding plots are shown in Fig. 8. The main lesson from these studies is that model 2 is absolutely stable in the entire parametrical region $0.2 \lesssim \kappa^{-1} B^{1 / 3} m_{a} / m_{\pi} \lesssim 0.95$, while model 1 is absolutely stable for $0.6 \lesssim \kappa^{-1} B^{1 / 3} m_{a}$ l

\footnotetext{
${ }^{11}$ In fact, there are many arguments suggesting that the cores of the neutron stars could be in the CS phase.
}

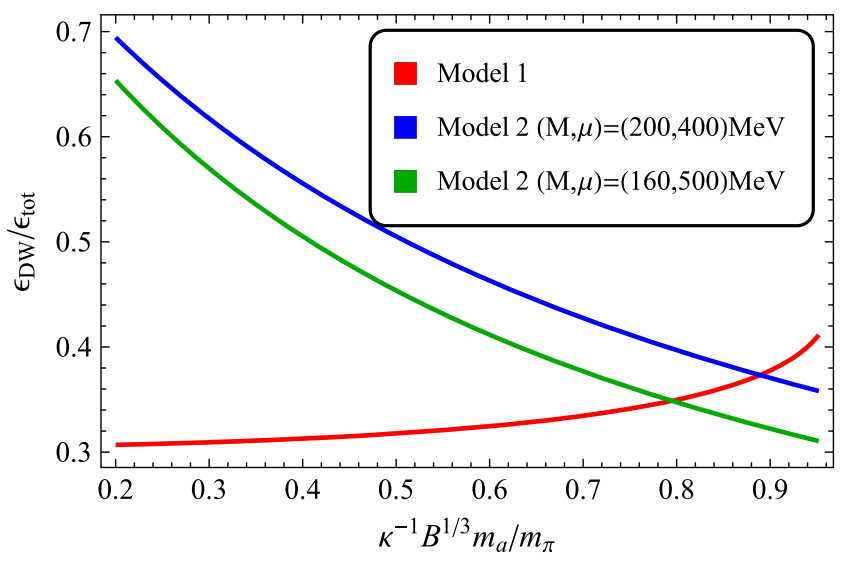

FIG. 9. Plot of $\epsilon_{\mathrm{DW}} / \epsilon_{\mathrm{tot}}$ vs $\kappa^{-1} B^{1 / 3} m_{a} / m_{\pi}$.

$m_{\pi} \lesssim 0.95$, and it becomes metastable for $0.2 \lesssim \kappa^{-1} B^{1 / 3} m_{a} I$ $m_{\pi} \lesssim 0.6$. This metastability (in contrast to the absolute stability) should not be a point of concern as discussed in the original paper [7] because it corresponds to a very long lifetime of the nuggets. This metastability region can be ignored for any practical purposes in our discussions in the present work.

For illustrative purposes we also present in Fig. 9 the plot for the domain wall contribution $\epsilon_{\mathrm{DW}} / \epsilon_{\mathrm{tot}}$ as a function of the same dimensionless parameter $\kappa^{-1} B^{1 / 3} m_{a} / m_{\pi}$. One can explicitly see from this plot that the energy related to the axion field represents a considerable portion of the nuggets' total energy representing approximately $1 / 3$ of the total energy for the model 1 (red curve). However, this contribution is very distinct from the conventional propagating axions (represented by $\Omega_{a}$ in this work) which are produced as a result of the misalignment mechanism. The energy of the axion field represented by $\epsilon_{\mathrm{DW}} / \epsilon_{\mathrm{tot}}$ cannot be easily released to space as the axions describing the axion domain wall are not on-shell axions. The corresponding energy in the form of propagating axions can only be released to the space when the nuggets get completely annihilated and the axions released, for example in the solar corona as discussed in $[33,34]$. It would be a major discovery if these axions could be observed by a CAST [63] or IAXO [62] type instrument.
[1] E. Witten, Cosmic separation of phases, Phys. Rev. D 30, 272 (1984).

[2] E. Farhi and R. L. Jaffe, Strange matter, Phys. Rev. D 30, 2379 (1984).

[3] A. De Rujula and S. L. Glashow, Nuclearites: A novel form of cosmic radiation, Nature (London) 312, 734 (1984).
[4] J. Madsen, Physics and astrophysics of strange quark matter, Lect. Notes Phys. 516, 162 (1999).

[5] Y. Aoki, G. Endrodi, Z. Fodor, S. D. Katz, and K. K. Szabo, The order of the quantum chromodynamics transition predicted by the standard model of particle physics, Nature (London) 443, 675 (2006). 
[6] C. Alcock and E. Farhi, Evaporation of strange matter in the early Universe, Phys. Rev. D 32, 1273 (1985).

[7] A. R. Zhitnitsky, "Nonbaryonic" dark matter as baryonic color superconductor, J. Cosmol. Astropart. Phys. 10 (2003) 010 .

[8] D. H. Oaknin and A. Zhitnitsky, Baryon asymmetry, dark matter and quantum chromodynamics, Phys. Rev. D 71, 023519 (2005).

[9] X. Liang and A. Zhitnitsky, Axion field and the quark nugget's formation at the QCD phase transition, Phys. Rev. D 94, 083502 (2016).

[10] S. Ge, X. Liang, and A. Zhitnitsky, Cosmological CP-odd axion field as the coherent Berry's phase of the Universe, Phys. Rev. D 96, 063514 (2017).

[11] R. D. Peccei and H. R. Quinn, Constraints imposed by $C P$ conservation in the presence of instantons, Phys. Rev. D 16, 1791 (1977); S. Weinberg, A New Light Boson?, Phys. Rev. Lett. 40, 223 (1978); F. Wilczek, Problem of Strong $P$ and $T$ Invariance in the Presence of Instantons, Phys. Rev. Lett. 40, 279 (1978).

[12] J. E. Kim, Weak-Interaction Singlet and Strong CP Invariance, Phys. Rev. Lett. 43, 103 (1979); M. A. Shifman, A. I. Vainshtein, and V. I. Zakharov, Can confinement ensure natural CP invariance of strong interactions?, Nucl. Phys. B166, 493 (1980).

[13] M. Dine, W. Fischler, and M. Srednicki, A simple solution to the strong CP problem with a harmless axion, Phys. Lett. 104B, 199 (1981); A. R. Zhitnitsky, Yad. Fiz. 31, 497 (1980) [Sov. J. Nucl. Phys. 31, 260 (1980)].

[14] K. van Bibber and L. J. Rosenberg, Ultrasensitive searches for the axion, Phys. Today 59, No. 8, 30 (2006).

[15] S. J. Asztalos, L. J. Rosenberg, K. van Bibber, P. Sikivie, and K. Zioutas, Searches for astrophysical and cosmological axions, Annu. Rev. Nucl. Part. Sci. 56, 293 (2006).

[16] P. Sikivie, Axion cosmology, Lect. Notes Phys. 741, 19 (2008).

[17] G. G. Raffelt, Astrophysical axion bounds, Lect. Notes Phys. 741, 51 (2008).

[18] P. Sikivie, Dark matter axions, Int. J. Mod. Phys. A 25, 554 (2010).

[19] L. J. Rosenberg, Dark-matter QCD-axion searches, Proc. Natl. Acad. Sci. U.S.A. 112, 12278 (2015).

[20] D. J. E. Marsh, Axion cosmology, Phys. Rep. 643, 1 (2016).

[21] P. W. Graham, I. G. Irastorza, S. K. Lamoreaux, A. Lindner, and K. A. van Bibber, Experimental searches for the axion and axion-like particles, Annu. Rev. Nucl. Part. Sci. 65, 485 (2015).

[22] A. Ringwald, Alternative dark matter candidates: Axions, in Proceedings of the Neutrino Oscillation Workshop (NOW2016), Otranto (Lecce, Italy) (2016) [arXiv:1612.08933].

[23] A. Zhitnitsky, Cold dark matter as compact composite objects, Phys. Rev. D 74, 043515 (2006).

[24] M. M. Forbes and A. R. Zhitnitsky, Diffuse x-rays: Directly observing dark matter?, J. Cosmol. Astropart. Phys. 01 (2008) 023.

[25] M. M. Forbes and A. R. Zhitnitsky, WMAP haze: Directly observing dark matter?, Phys. Rev. D 78, 083505 (2008).

[26] M. M. Forbes, K. Lawson, and A. R. Zhitnitsky, Electrosphere of macroscopic "quark nuclei": A source for diffuse
MeV emissions from dark matter, Phys. Rev. D 82, 083510 (2010).

[27] K. Lawson and A. R. Zhitnitsky, Isotropic radio background from quark nugget dark matter, Phys. Lett. B 724, 17 (2013).

[28] D. H. Oaknin and A. R. Zhitnitsky, $511 \mathrm{keV}$ Photons from Color Superconducting Dark Matter, Phys. Rev. Lett. 94, 101301 (2005).

[29] A. Zhitnitsky, Width of the $511 \mathrm{keV}$ line from the bulge of the galaxy, Phys. Rev. D 76, 103518 (2007).

[30] K. Lawson and A. R. Zhitnitsky, Diffuse cosmic gammarays at 1-20 MeV: A trace of the dark matter?, J. Cosmol. Astropart. Phys. 01 (2008) 022.

[31] K. Lawson and A. R. Zhitnitsky, Quark (anti)nugget dark matter, in Snowmass 2013 e-Proceedings - Cosmic Frontier Workshop (2013) [arXiv:1305.6318].

[32] D. M. Jacobs, G. D. Starkman, and B. W. Lynn, Macro dark matter, Mon. Not. R. Astron. Soc. 450, 3418 (2015).

[33] A. Zhitnitsky, Solar extreme UV radiation and quark nugget dark matter model, J. Cosmol. Astropart. Phys. 10 (2017) 050 .

[34] A. Zhitnitsky, Solar flares and the axion quark nugget dark matter model, arXiv:1801.01509.

[35] J. Preskill, M. B. Wise, and F. Wilczek, Cosmology of the invisible axion, Phys. Lett. 120B, 127 (1983); L. Abbott and P. Sikivie, A Cosmological Bound on the Invisible Axion, Phys. Lett. 120B, 133 (1983); M. Dine and W. Fischler, The not-so-harmless axion, Phys. Lett. 120B, 137 (1983).

[36] S. Chang, C. Hagmann, and P. Sikivie, Studies of the motion and decay of axion walls bounded by strings, Phys. Rev. D 59, 023505 (1998).

[37] T. Hiramatsu, M. Kawasaki, K. Saikawa, and T. Sekiguchi, Production of dark matter axions from collapse of stringwall systems, Phys. Rev. D 85, 105020 (2012); Erratum, Phys. Rev. D 86, 089902(E) (2012).

[38] M. Kawasaki, K. Saikawa, and T. Sekiguchi, Axion dark matter from topological defects, Phys. Rev. D 91, 065014 (2015).

[39] L. Fleury and G. D. Moore, Axion dark matter: Strings and their cores, J. Cosmol. Astropart. Phys. 01 (2016) 004.

[40] V. B. Klaer and G. D. Moore, The dark-matter axion mass, J. Cosmol. Astropart. Phys. 11 (2017) 049.

[41] L. Visinelli and P. Gondolo, Dark matter axions revisited, Phys. Rev. D 80, 035024 (2009).

[42] S. Borsanyi et al., Calculation of the axion mass based on high-temperature lattice quantum chromodynamics, Nature (London) 539, 69 (2016).

[43] D. T. Son, M. A. Stephanov, and A. R. Zhitnitsky, Domain Walls of High-Density QCD, Phys. Rev. Lett. 86, 3955 (2001).

[44] T. Kojo, P. D. Powell, Y. Song, and G. Baym, Phenomenological QCD equation of state for massive neutron stars, Phys. Rev. D 91, 045003 (2015).

[45] P. W. Gorham, Antiquark nuggets as dark matter: New constraints and detection prospects, Phys. Rev. D 86, 123005 (2012).

[46] P. W. Gorham and B. J. Rotter, Stringent neutrino flux constraints on antiquark nugget dark matter, Phys. Rev. D 95, 103002 (2017). 
[47] K. Lawson and A. R. Zhitnitsky, Solar neutrino spectrum of quark nugget dark matter, Phys. Rev. D 95, 063521 (2017).

[48] K. Lawson and A. R. Zhitnitsky, Quark nugget dark matter: Comparison with radio observations of nearby galaxies, Phys. Lett. B 757, 376 (2016).

[49] K. Lawson and A. Zhitnitsky, Quark nugget dark matter: No contradiction with $511 \mathrm{keV}$ line emission from dwarf galaxies, J. Cosmol. Astropart. Phys. 02 (2017) 049.

[50] A. Arvanitaki, M. Baryakhtar, and X. Huang, Discovering the QCD axion with black holes and gravitational waves, Phys. Rev. D 91, 084011 (2015).

[51] P. A. R. Ade et al. (BICEP2 and Planck Collaborations), Joint Analysis of BICEP2/Keck Array and Planck Data, Phys. Rev. Lett. 114, 101301 (2015).

[52] A. D. Linde, Generation of isothermal density perturbations in the inflationary Universe, Phys. Lett. 158B, 375 (1985).

[53] D. Seckel and M. S. Turner, "Isothermal" density perturbations in an axion-dominated inflationary universe, Phys. Rev. D 32, 3178 (1985).

[54] M. P. Hertzberg, M. Tegmark, and F. Wilczek, Axion cosmology and the energy scale of inflation, Phys. Rev. D 78, 083507 (2008).

[55] J. Hamann, S. Hannestad, G. G. Raffelt, and Y. Y. Y. Wong, Isocurvature forecast in the anthropic axion window, J. Cosmol. Astropart. Phys. 06 (2009) 022.

[56] T. Kobayashi, R. Kurematsu, and F. Takahashi, Isocurvature constraints and anharmonic effects on QCD axion dark matter, J. Cosmol. Astropart. Phys. 09 (2013) 032.

[57] C. Beck, Possible Resonance Effect of Axionic Dark Matter in Josephson Junctions, Phys. Rev. Lett. 111, 231801 (2013).
[58] C. Beck, Axion mass estimates from resonant Josephson junctions, Phys. Dark Universe 7-8, 6 (2015).

[59] C. Beck, Possible resonance effect of dark matter axions in SNS Josephson junctions, in Based on talk given at the EPS Conference on High Energy Physics, 5-12 July 2017 Venice (2017) [arXiv:1710.04299].

[60] S. Bertolucci, K. Zioutas, S. Hofmann, and M. Maroudas, The Sun and its planets as detectors for invisible matter, Phys. Dark Universe 17, 13 (2017).

[61] I. Stern, ADMX status, Proc. Sci., ICHEP2016 (2016) 198 [arXiv:1612.08296].

[62] E. Armengaud et al. (IAXO Collaboration), Conceptual design of the International Axion Observatory (IAXO), J. Instrum. 9, T05002 (2014).

[63] V. Anastassopoulos et al. (CAST Collaboration), New CAST limit on the axion-photon interaction, Nat. Phys. 13, 584 (2017).

[64] G. Rybka, A. Wagner, A. Brill, K. Ramos, R. Percival, and K. Patel, Search for dark matter axions with the Orpheus experiment, Phys. Rev. D 91, 011701 (2015).

[65] A. Caldwell, G. Dvali, B. Majorovits, A. Millar, G. Raffelt, J. Redondo, O. Reimann, F. Simon, and F. Steffen (MADMAX Working Group), Dielectric Haloscopes: A New Way to Detect Axion Dark Matter, Phys. Rev. Lett. 118, 091801 (2017).

[66] B. T. McAllister, G. Flower, J. Kruger, E. N. Ivanov, M. Goryachev, J. Bourhill, and M.E. Tobar, The ORGAN experiment: An axion haloscope above $15 \mathrm{GHz}$, Phys. Dark Universe 18, 67 (2017).

[67] C. Cao and A. Zhitnitsky, Axion detection via topological Casimir effect, Phys. Rev. D 96, 015013 (2017).

[68] G. Cantatore, Investigating short-distance interactions at submicron scales, http://indico.ibs.re.kr/event/137/contribution/ 33/material/slides/0.pdf. 